\title{
Publicité alimentaire destinée aux enfants : examen de la portée de l'exposition, du pouvoir et des répercussions selon le contexte
}

\author{
Rachel Prowse, B. Sc., diététiste
}

Cet article a fait l'objet d'une évaluation par les pairs.

\section{Résumé}

Introduction : La publicité alimentaire a des répercussions sur les connaissances et les comportements alimentaires des enfants ainsi que sur leur santé. La réglementation actuelle au Canada est axée sur la restriction des aspects promotionnels de la publicité alimentaire, mais elle accorde peu d'attention, voire aucune, à l'endroit où se fait l'expérience alimentaire des enfants. Il est essentiel de comprendre où s'inscrit la publicité alimentaire dans le quotidien des enfants pour pouvoir les protéger. Notre étude fait état de la littérature sur la publicité alimentaire destinée aux enfants au Canada en fonction du contexte.

Méthodologie : L'auteure a consulté des bases de données pour trouver des travaux de recherche canadiens portant sur l'exposition des enfants et des jeunes (de 2 à 17 ans) à la publicité alimentaire, sur le pouvoir et les répercussions que celle-ci peut avoir sur les enfants dans différents contextes et sur la façon dont la réglementation actuelle peut en atténuer l'effet sur les enfants. Ont été sélectionnées les études en anglais, examinées par des pairs et publiées entre 2000 et 2016.

Résultats : Vingt-cinq études se sont intéressées à l'exposition des enfants à la publicité alimentaire et au pouvoir ou aux répercussions qu'elle peut avoir sur ceux-ci à la maison (par la télévision ou Internet) $(n=12)$, dans les écoles publiques $(n=1)$, dans les épiceries $(n=8)$, dans les restaurants rapides $(n=2)$ et en général $(n=2)$. Les tendances de la recherche révèlent que les aliments malsains ciblent les enfants par différentes techniques promotionnelles, qui se chevauchent selon les contextes. Il existe plusieurs lacunes en matière de recherche dans ce domaine, ce qui donne un portrait incomplet et potentiellement sous-estimé de la publicité alimentaire destinée aux enfants au Canada. Les données probantes disponibles indiquent que les approches canadiennes actuelles n'ont pas permis de réduire l'exposition des enfants à la publicité alimentaire ni le pouvoir de celleci dans ces contextes, à l'exception de certaines influences positives de la réglementation officielle au Québec.

Conclusion : Les contextes dans lesquels les enfants consomment des aliments, en achètent ou apprennent à les connaître les exposent à une publicité puissante qui, souvent, porte sur des aliments malsains. Les données probantes indiquent que la " place » pourrait être une composante de marketing importante à inclure dans les politiques publiques afin de protéger les enfants de la publicité sur les aliments malsains d'une façon générale. Les organisations et les collectivités peuvent participer aux interventions de promotion de la santé en fonction des contextes en élaborant leurs propres politiques de marketing pour s'attaquer à la promotion et à la distribution de boissons et d'aliments malsains.

Mots clés : publicité alimentaire, obésité infantile, santé publique

\section{Introduction}

Le développement des enfants se fait dans les situations de la vie quotidienne ${ }^{1}$. Les endroits où ils vivent, apprennent et jouent constituent des facteurs déterminants pour leur santé actuelle et future ${ }^{2}$. La Charte d'Ottawa pour la promotion de la santé souligne d'ailleurs l'importance des situations de la vie quotidienne dans la
Points saillants

- Les situations de la vie quotidienne des enfants constituent des contextes dans lesquels il est important de restreindre la publicité sur les aliments malsains.

- La recherche au Canada montre que les enfants et les jeunes (de 2 à 17 ans) sont exposés à la publicité alimentaire à la maison, dans les écoles et dans les supermarchés. L'exposition générale est probablement sous-estimée.

- Des techniques de marketing puissantes sont souvent utilisées pour promouvoir des aliments moins sains auprès des enfants.

- Plusieurs expositions à la publicité sur les aliments malsains dans différents contextes peuvent nuire à la manière dont la culture alimentaire des enfants est façonnée.

- Même si des recherches plus approfondies sont nécessaires, les données probantes actuelles sont suffisantes pour prouver que les gouvernements et les collectivités ont à étudier toutes les composantes du marketing (produit, promotion, prix et distribution) pour protéger les enfants du pouvoir dans leur vie quotidienne de la publicité sur les aliments malsains.

prévention des maladies ${ }^{3}$. À cette fin, l'Organisation mondiale de la santé recommande d'éliminer la publicité sur les boissons et les aliments malsains dans les endroits où les enfants se rassemblent ${ }^{4}$. Par ailleurs, la distribution constitue un facteur déterminant pour les spécialistes du marketing : il s'agit de l'une des quatre composantes du marketing connues sous le nom des « $4 \mathrm{P}$ » : produit, promotion, prix 
et place (ou distribution). Les entreprises mélangent les « $4 \mathrm{P}$ » de façon stratégique pour atteindre leur public cible efficacement et influencer les attitudes et les comportements ${ }^{5}$.

La publicité alimentaire a des répercussions sur les connaissances, les préférences et les comportements alimentaires des enfants ainsi que sur leur santét. Les facteurs qui favorisent une mauvaise alimentation sont préoccupants, car, selon Statistique Canada, le quart des calories consommées par les jeunes Canadiens de 4 à 18 ans proviennent souvent des " autres aliments » (p. ex. aliments à limiter selon le Guide alimentaire canadien), en particulier les boissons gazeuses, les boissons aux fruits, le chocolat et les croustilles ${ }^{7}$. Plus de la moitié des enfants au Canada consomment moins de cinq portions de fruits et de légumes par jour ${ }^{8}$. Les répercussions de la publicité alimentaire sur les préférences et les comportements alimentaires des enfants dépendent de leur exposition aux messages publicitaires et du pouvoir que ces derniers exercent sur eux. L'exposition est définie comme étant « la portée et la fréquence du message publicitaire ", tandis que le pouvoir désigne « la conception, l'exécution et le contenu créatifs du message publicitaire $»^{9, p .11}$ [traduction].

La publicité alimentaire destinée aux enfants est actuellement " régie » par trois mécanismes principaux au Canada (tableau 1) : (1) la réglementation officielle au Québec (Loi sur la protection du consommateur du Québec [LPCQ] ${ }^{10}$ ), (2) l'autoréglementation volontaire de l'industrie alimentaire (Initiative canadienne pour la publicité sur les aliments et les boissons destinée aux enfants $[\mathrm{IPE}]^{11}$ ) et (3) l'autoréglementation de l'industrie de la radiodiffusion (Code de la publicité radiotélévisée destinée aux enfants ${ }^{12}$ ). De plus, en 2016, la ministre de la Santé du Canada a annoncé la création de nouvelles dispositions légales fédérales sur la publicité alimentaire ${ }^{13}$. Les politiques alimentaires des écoles peuvent également réglementer la publicité alimentaire destinée aux enfants. Les politiques provinciales et territoriales actuelles ont cependant tendance à porter sur l'approvisionnement alimentaire, demeurant limitées et manquant de cohérence dans leur façon d'aborder la publicité alimentaire (tableau 1).
Les règlements actuels et en préparation visent à régir l'exposition à la publicité alimentaire destinée aux enfants en restreignant la quantité et l'utilisation de techniques promotionnelles persuasives (dont il est question dans la section "Résultats » de cet article). Malheureusement, cette approche tient peu compte de la distribution, pourtant une composante clé des stratégies employées par les spécialistes du marketing et dans les interventions de promotion de la santé14 - à l'exception de l'IPE, qui restreint dans une certaine mesure la publicité dans les écoles primaires ${ }^{15}$ * . On peut donc s'attendre à ce que les règlements qui négligent la distribution n'aient pas d'impact décisif sur l'exposition des enfants à la publicité alimentaire ou sur le pouvoir qu'elle exerce sur eux. On interprète souvent à tort la distribution comme étant la localisation des messages publicitaires, alors que celle-ci fait plutôt partie de la promotion ${ }^{16}$. Du point de vue du marketing, il serait plus exact de définir la distribution comme étant le lieu où les comportements ont lieu, ou bien celui où les biens et les services qui leur sont reliés sont acquis ${ }^{5}$. Dans le contexte de la publicité alimentaire, la distribution peut ainsi désigner le lieu où nous consommons les aliments, celui où nous les achetons et celui où nous apprenons à les connaître.

L'une des considérations stratégiques de la réglementation proposée au Canada ${ }^{17}$ concerne justement les contextes dans lesquels les enfants sont visés par la publicité. Toutefois, aucun travail de recherche ne s'y est encore intéressé. Il est pourtant essentiel, si l'on veut élaborer des politiques efficaces, de comprendre la publicité alimentaire dans le contexte dans lequel les enfants l'expérimentent. Notre étude vise, grâce à une approche fondée sur le contexte ${ }^{18}$, à explorer les lieux où les enfants peuvent être exposés à la publicité alimentaire. Elle porte sur l'étendue de leur exposition et le pouvoir de la publicité alimentaire en fonction du contexte, sur l'influence de la réglementation officielle (LPCQ) et de l'autoréglementation volontaire (IPE) sur l'exposition et le pouvoir ${ }^{\dagger}$ et enfin sur les répercussions de la publicité alimentaire sur les attitudes, les perceptions et les comportements des enfants canadiens.

\section{Méthodologie}

En juillet 2015, l'auteure a consulté de manière exhaustive huit bases de données en santé, en psychologie et en affaires (tableau 2) répertoriées par un documentaliste spécialisé en recherche, afin d'étudier l'exposition à la publicité alimentaire destinée aux enfants au Canada, le pouvoir et les répercussions qu'elle a sur eux et l'influence de la réglementation. Elle a mis à jour ces résultats de recherche en septembre 2016. Toutes les références ont été importées dans un gestionnaire de références en ligne. L'auteure a choisi les articles en fonction de critères d'inclusion définis à priori (tableau 2) par une analyse systématique des titres, des résumés et du texte intégral (figure 1). Après avoir examiné les titres et les résumés, elle a consulté trois chercheurs canadiens experts dans le domaine afin de relever les lacunes dans la recherche et de confirmer l'exhaustivité des résultats. Ces chercheurs ont présenté 21 nouveaux éléments, dont seulement 4 correspondaient aux critères d'inclusion $^{34-37}$ (figure 1). Cette étude de portée a été limitée aux études en anglais évaluées par des pairs et utilisant des données canadiennes. Deux articles en français $^{38,39}$ ont été exclus car aucun expert s'exprimant couramment dans cette langue n'a été en mesure de les examiner. L'auteure a lu toutes les études et en a extrait les données.

\section{Résultats}

Vingt-cinq articles ont répondu aux critères d'inclusion (figure 1). La littérature portait sur l'exposition à la publicité alimentaire destinée aux enfants au Canada et sur le pouvoir ou les répercussions qu'elle a sur ceux-ci en général $^{36,40}$, à la télévision ${ }^{34,41-48}$, sur Internet ${ }^{49-51}$, dans les écoles publiques ${ }^{52}$, sur les emballages de produits dans les épiceries $^{35,37,53-58}$ et dans les restaurants rapides $^{59,60}$ (tableau 3). La plupart des articles étaient fondés sur des études transversales $(n=14)^{34,37,42-45,49-56}$. Deux articles s'intéressaient aux répercussions de la $\mathrm{LPCQ}^{43,44}$, et quatre portaient sur les répercussions de l'IPE sur l'exposition à la publicité alimentaire et sur le pouvoir qu'elle exerce $^{45-47,56}$. Le tableau 4 présente une synthèse de l'influence des règlements sur l'exposition et le pouvoir en fonction du contexte. Neuf études traitaient de l'incidence

* Le prix, une autre composante des « 4 P », n'est pas défini non plus dans la réglementation sur le marketing. Cependant, cette question dépasse la portée de cette étude.
† Les chercheurs n'ont pas évalué le Code de la publicité radiotélévisée destinée aux enfants. Par conséquent, cette étude ne porte que sur l'influence de l'IPE et de la LPCQ. 
TABLEAU 1

Types de réglementation en matière de publicité alimentaire destinée aux enfants au Canada

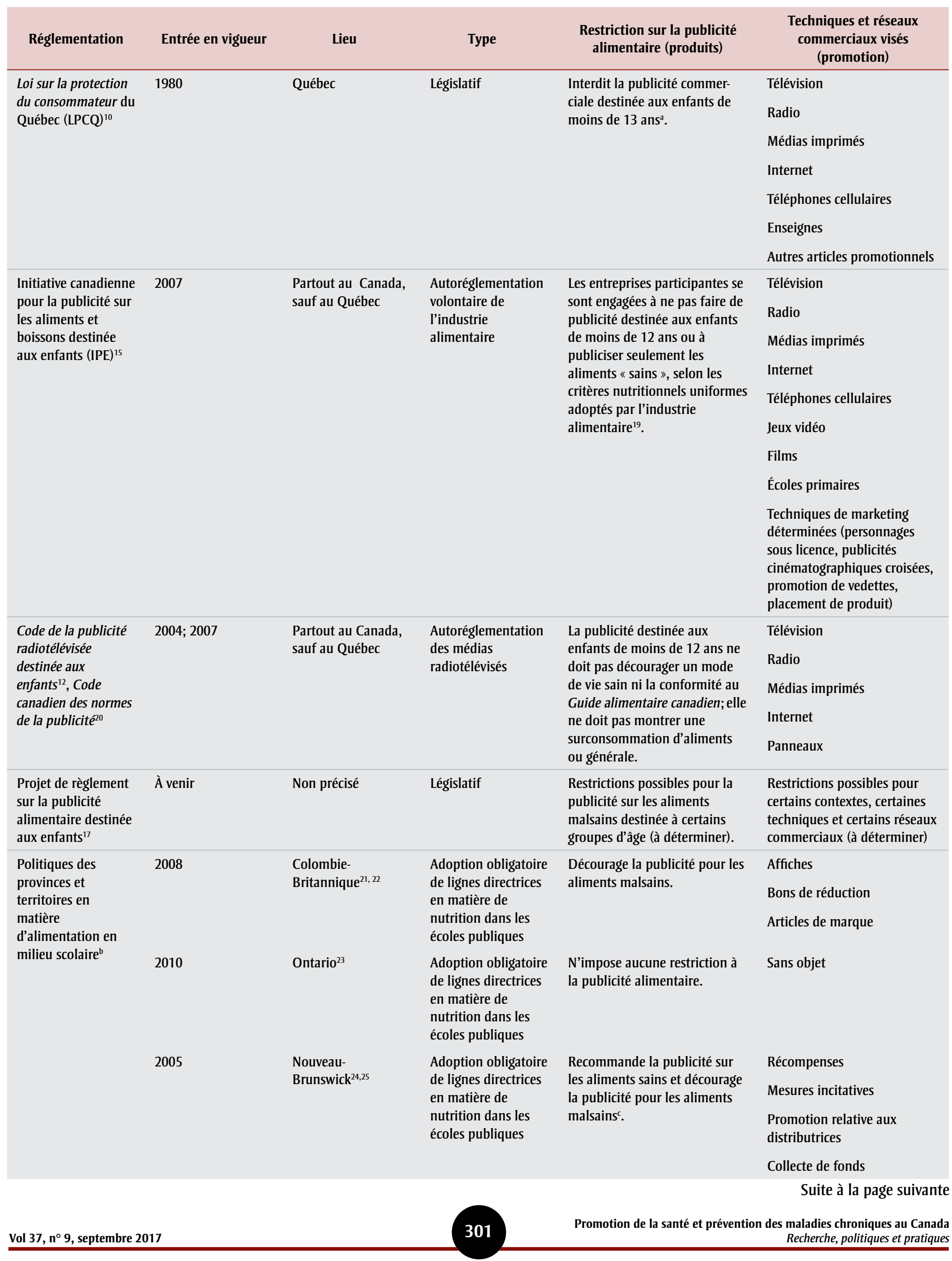


TABLEAU 1 (suite)

Types de réglementation en matière de publicité alimentaire destinée aux enfants au Canada

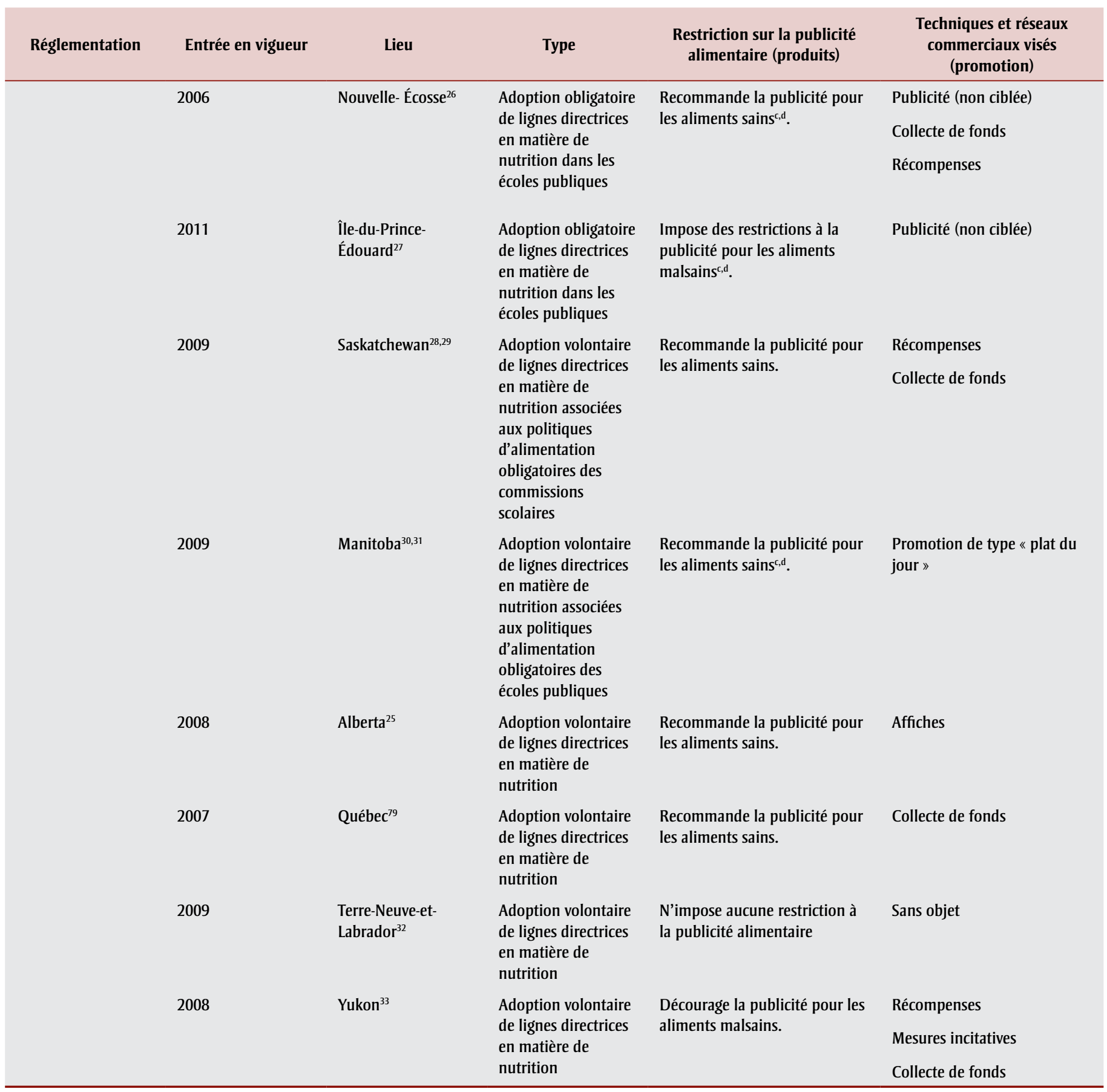

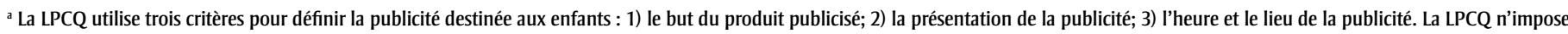
pas de restriction explicite à la publicité dans les écoles ou au point de vente, mais celle-ci peut être interdite si on applique les critères ${ }^{10}$.

${ }^{\text {b }}$ Aucune politique n'était accessible au public dans les Territoires du Nord-Ouest et au Nunavut.

' Comprend les énoncés sur le prix des aliments.

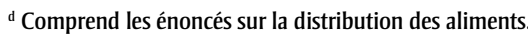


TABLEAU 2

Examen de la portée de la publicité alimentaire destinée aux enfants au Canada : critères et processus de recherche systématique

\begin{tabular}{|c|c|}
\hline \multirow[t]{7}{*}{ Critères d'inclusion } & Anglais \\
\hline & Données canadiennes \\
\hline & Publication entre janvier 2000 et septembre 2016 \\
\hline & Recherche originale \\
\hline & $\begin{array}{l}\text { Données probantes portant sur l'exposition des enfants et des jeunes (de } 2 \text { à } \\
17 \text { ans) à la publicité alimentaire, sur le pouvoir ou les répercussions que la } \\
\text { publicité alimentaire peut avoir sur les enfants, ou sur l'influence de la } \\
\text { réglementation canadienne sur la publicité alimentaire }\end{array}$ \\
\hline & $\begin{array}{l}\text { Dans les données probantes portant sur l'exposition, le pouvoir et la réglemen- } \\
\text { tation, le contexte doit être défini. }\end{array}$ \\
\hline & $\begin{array}{l}\text { Dans les données probantes portant sur les répercussions, le contexte doit être } \\
\text { clairement défini, ou les répercussions collectives de la publicité alimentaire } \\
\text { dans l'ensemble des contextes doivent être étudiées. }\end{array}$ \\
\hline \multirow[t]{4}{*}{ Critères d'exclusion } & Littérature grise \\
\hline & $\begin{array}{l}\text { Données probantes portant sur les nouveau-nés et les nourrissons (moins de } \\
2 \text { ans) }\end{array}$ \\
\hline & Données probantes portant sur les parents seulement \\
\hline & Commentaires sur les interventions stratégiques \\
\hline Chaîne de recherche & $\begin{array}{l}\text { (food OR beverage OR diet OR nutrition [TIAB]), AND (marketing OR } \\
\text { advertis*[TIAB]), AND (child* OR youth OR teen OR adolescen*[TIAB]), AND } \\
\left(\text { Canad*[TIAB]) }^{*} \text {. }\right.\end{array}$ \\
\hline $\begin{array}{l}\text { Bases de données } \\
\text { consultées }\end{array}$ & $\begin{array}{l}\text { ABI/INFORM Complete, CBCA Complete, CINAHL, MEDLINE, ProQuest } \\
\text { Dissertation \& Theses, PsycINFO, Scopus, Web of Science Core }\end{array}$ \\
\hline
\end{tabular}

de la publicité alimentaire sur les attitudes, les préférences et les comportements alimentaires : trois ont fait appel à des méthodes expérimentales ${ }^{48,59,60}$, une a utilisé des méthodes transversales ${ }^{49}$ et cinq ont eu recours à des méthodes qualitatives ${ }^{35,36,40,57,58}$.

\section{Exposition à la publicité alimentaire destinée aux enfants au Canada et pouvoir de celle-ci}

\section{Exposition à la publicité alimentaire à la maison : télévision}

Six articles portaient sur l'exposition à la publicité alimentaire télévisée ${ }^{34,41-45}$. Dans ces études, l'exposition a été mesurée par la proportion d'annonces télévisées axées sur des aliments (tout type d'aliments et aliments malsains) et la fréquence de ces annonces par heure par chaîne.

Un cinquième des annonces enregistrées sur trois chaînes populaires pour enfants au Canada en 2007-2008 concernait des aliments (données non publiées de Kelly et al. ${ }^{34}$ ). En étudiant les 30 premières heures de télévision (chaînes généralistes et les chaînes pour enfants) regardées par les enfants de 10 à 12 ans en Ontario et au
Québec en 2009, Potvin Kent et al. ont constaté que $24 \%$ à $27 \%$ des annonces regardées par les enfants portaient sur des aliments ${ }^{43}$.

Les études ont fait état de fréquences d'annonces alimentaires différentes : entre trois et sept annonces par heure par chaîne $^{34,41,47}$ (données non publiées de Kelly et $\left.a l .{ }^{34}\right)$. Cette variabilité est sans doute due aux différences de méthodologie entre études, notamment à une hétérogénéité dans le nombre et le type de chaînes enregistrées, dans les heures et dans le nombre de jours enregistrés ainsi que dans le lieu et dans les dates de collecte des données.

On a évalué l'exposition aux annonces télévisées sur des aliments malsains en déterminant la proportion d'aliments à haute teneur en énergie, en gras, en sucre ou en sel annoncés ${ }^{34,42,44}$. Selon Kelly et al. ${ }^{34}, 80 \%$ des annonces alimentaires diffusées sur les chaînes pour enfants concernaient des aliments à haute teneur en gras, en sodium ou en énergie autres que des " aliments de base ». À l'aide du système axé sur le profil nutritionnel du RoyaumeUni, Adams et al. ${ }^{42}$ ont constaté que $66 \%$ des annonces alimentaires diffusées sur les chaînes généralistes au Canada proposaient des aliments "moins sains ». À l'aide du même système de profil nutritionnel, Potvin Kent et al. ${ }^{44}$ ont observé que $88 \%$ des annonces alimentaires regardées par les enfants au Canada faisaient la promotion d'aliments " moins sains ".

\section{Influence de la réglementation sur l'exposition}

Potvin Kent et al. ont fait des recherches en 2009 sur les répercussions de la réglementation officielle ${ }^{43}$ et en 2011 sur celles de la réglementation volontaire de l'industrie $^{46}$ au Canada. Ils ont montré que ni l'une ni l'autre n'était associée à une réduction de l'exposition des enfants à la publicité alimentaire télévisée. Plus précisément, il semblerait que les enfants francophones au Québec et les enfants anglophones au Québec et en Ontario aient été exposés à la même fréquence d'annonces alimentaires par heure par chaîne ${ }^{43}$. Potvin Kent et Wanless $^{47}$ ont estimé que, depuis l'introduction de l'IPE, l'exposition générale des enfants aux annonces alimentaires télévisées avait augmenté de $6 \%$ à Vancouver et de $17 \%$ à Toronto entre 2006 et 2011. Même si les annonces alimentaires diffusées sur les chaînes pour enfants par des entreprises de l'IPE ont diminué de $24 \%$ entre 2006 et 2011, le même type d'annonces diffusées par des entreprises autres que celles de l'IPE a augmenté de $76 \%{ }^{46}$.

De légères améliorations dans la qualité nutritionnelle des aliments annoncés ont été associées à la $\mathrm{LPCQ}^{43}$, mais pas à l'IPE $^{46}$. On a observé que le nombre d'annonces pour des aliments " moins sains » regardées par les enfants était beaucoup moins élevé sur les chaînes de télévision francophones au Québec que sur les chaînes de télévision anglophones en Ontario $^{44}$. Cependant, $81 \%$ des annonces regardées par les enfants sur les chaînes de télévision francophones au Québec touchaient des aliments "moins sains ". Par ailleurs, il n'y a eu aucun changement important dans la proportion d'aliments " moins sains " annoncés par les entreprises de l'IPE entre 2006 et $2011^{46}$.

\section{Pouvoir de la publicité alimentaire à la} maison : télévision

Le pouvoir de la publicité alimentaire a été évalué par la fréquence des annonces alimentaires ciblant les enfants et le recours à des techniques promotionnelles puissantes. Pendant les heures de grande écoute (de 19 h à 23 h), 7 \% des annonces alimentaires étaient particulièrement attrayantes 
FIGURE 1

Diagramme illustrant la stratégie de recherche systématique

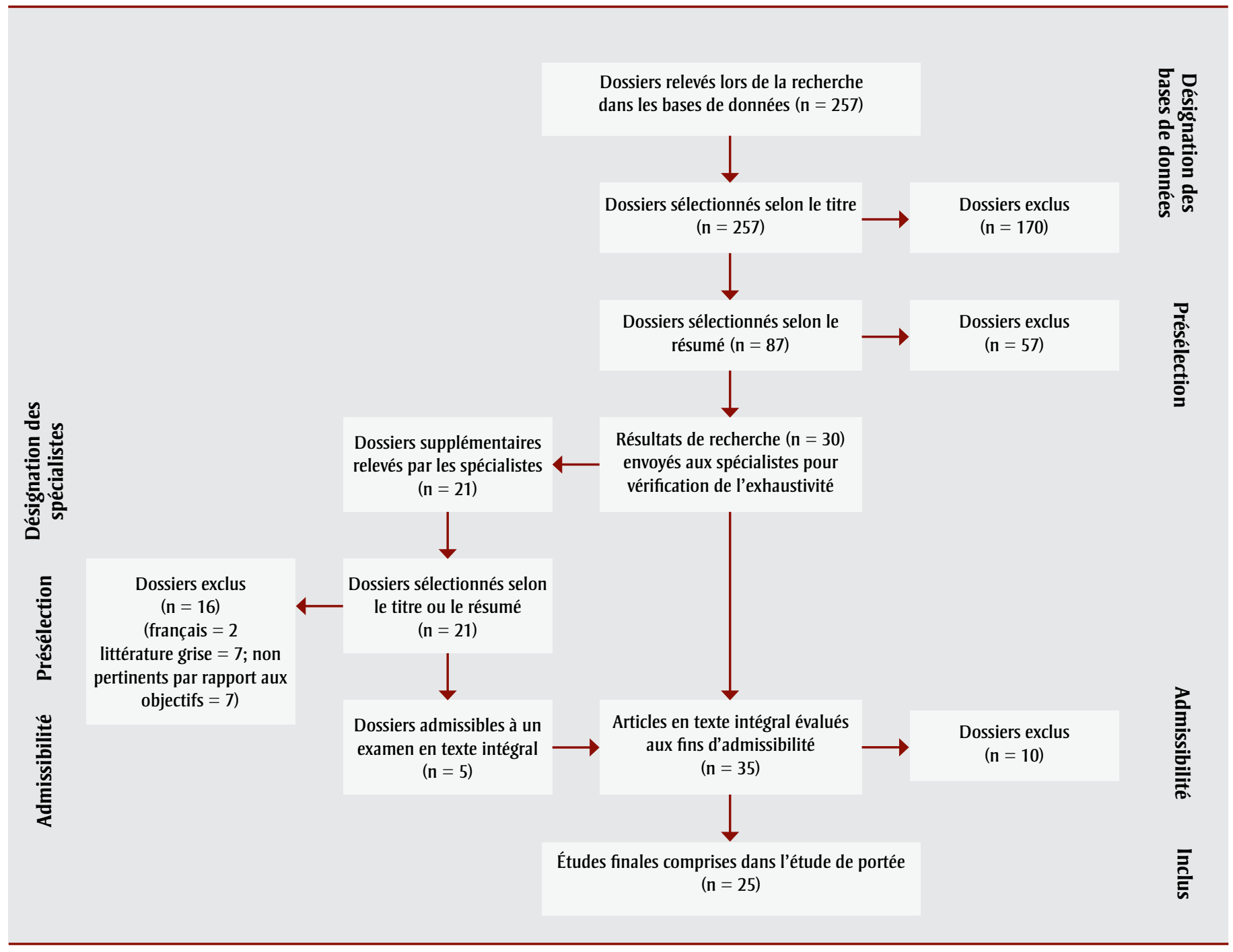

pour les enfants et les jeunes (de 2 à 17 ans) en $2006^{42}$. Sur les chaînes télévisées regardées par les enfants (de 10 à 12 ans) francophones au Québec en 2009, seulement $30 \%$ des annonces alimentaires ciblaient les enfants, contre $76 \%$ des annonces regardées par les enfants (de 10 à 12 ans) anglophones au Québec et $65 \%$ en Ontario $^{43}$. En 2011, environ le quart des annonces alimentaires diffusées par les entreprises de l'IPE et les autres entreprises sur les chaînes spécialisées pour enfants ciblait les enfants et les adolescents ${ }^{46}$.

Diverses techniques de marketing étaient utilisées dans les annonces alimentaires télévisées, notamment des primes (comme les cadeaux publicitaires ou les coupons), des personnages promotionnels et des éléments amusants et invoquant la santé ${ }^{34,46}$. Les aliments annoncés à l'aide de ces techniques puissantes étaient souvent malsains ${ }^{34,46}$.
Par exemple, Kelly et al. ${ }^{34}$ ont montré que presque $100 \%$ des annonces alimentaires télévisées ayant recours à des personnages promotionnels sur les chaînes pour enfants en 2007 et en 2008 au Canada concernaient des aliments autres que les " aliments de base ", ce qui était le cas de seulement $80 \%$ des annonces dans l'ensemble.

\section{Influence de la réglementation sur le pouvoir}

De légères améliorations ayant trait au pouvoir des annonces alimentaires semblent associées à la $\mathrm{LPCQ}^{43}$, mais pas à l'IPE ${ }^{46}$. En 2009, la LPCQ a été associée à un nombre inférieur d'annonces alimentaires ciblant les enfants francophones au Québec, mais n'a pas permis de protéger complètement tous les enfants au Québec, puisque les enfants anglophones au Québec regardent du contenu télévisuel provenant de l'extérieur du Québec, et qui n'est donc pas assujetti à la loi du Québec ${ }^{43}$. Dans l'ensemble, il n'y a eu aucun changement dans la fréquence des annonces alimentaires ciblant les enfants passées par des entreprises de l'IPE et les autres entreprises entre 2006 et $2011^{46}$. En fait, certaines données probantes indiquent que la situation a empiré, puisque le nombre d'annonces proposant des aliments malsains et ciblant les enfants était plus élevé en 2011 qu'en $2006^{46}$. Par exemple, entre 2006 et 2011, l'utilisation de personnages amusants et de personnages reproduits sous licence pour annoncer des produits "moins sains » a augmenté de respectivement $38 \%$ et $234 \%$, parmi les entreprises de l'IPE ${ }^{46}$.

\section{Exposition à la publicité alimentaire à la maison : Internet}

Deux études s'intéressant à la publicité alimentaire sur Internet au Canada ont traité 


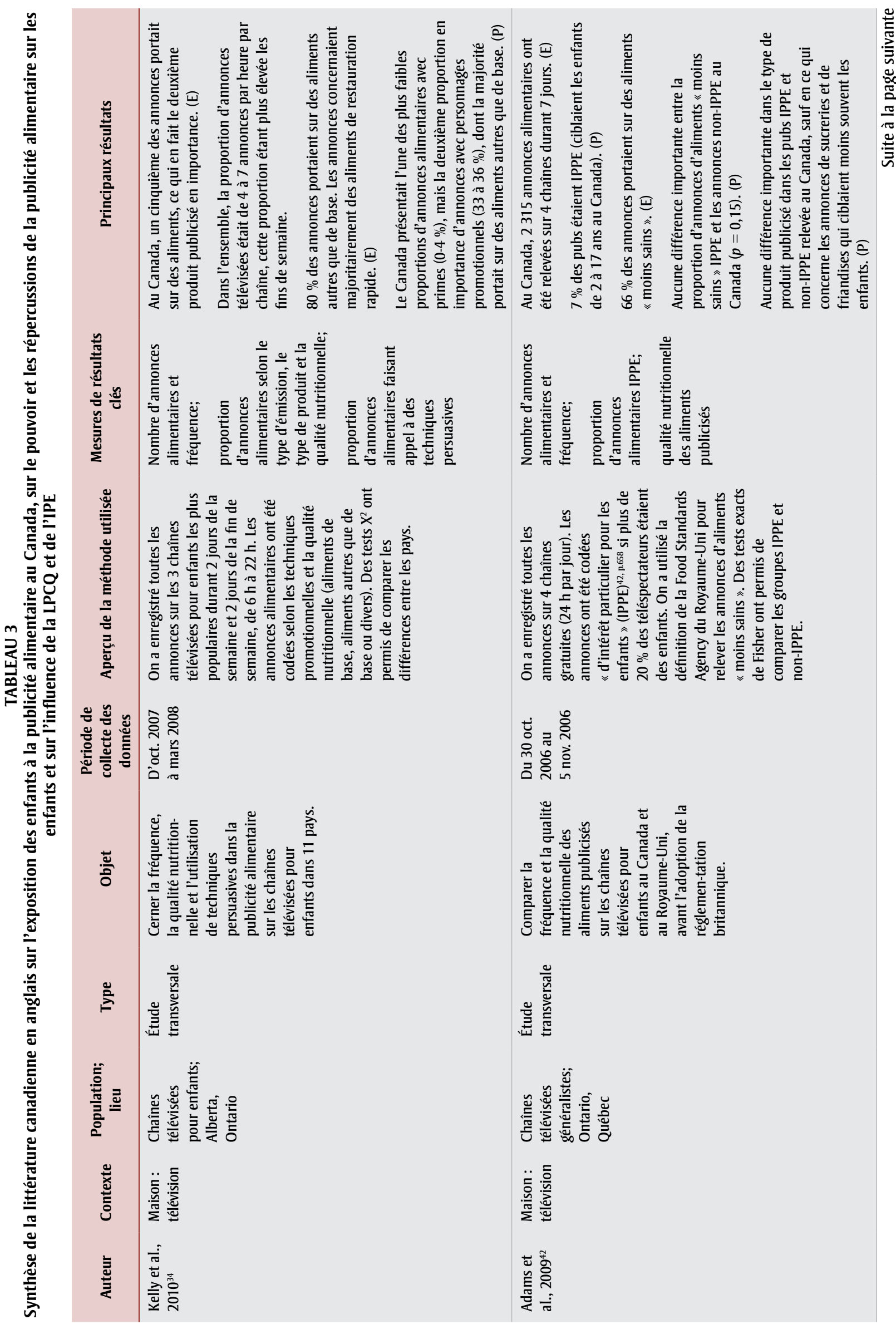




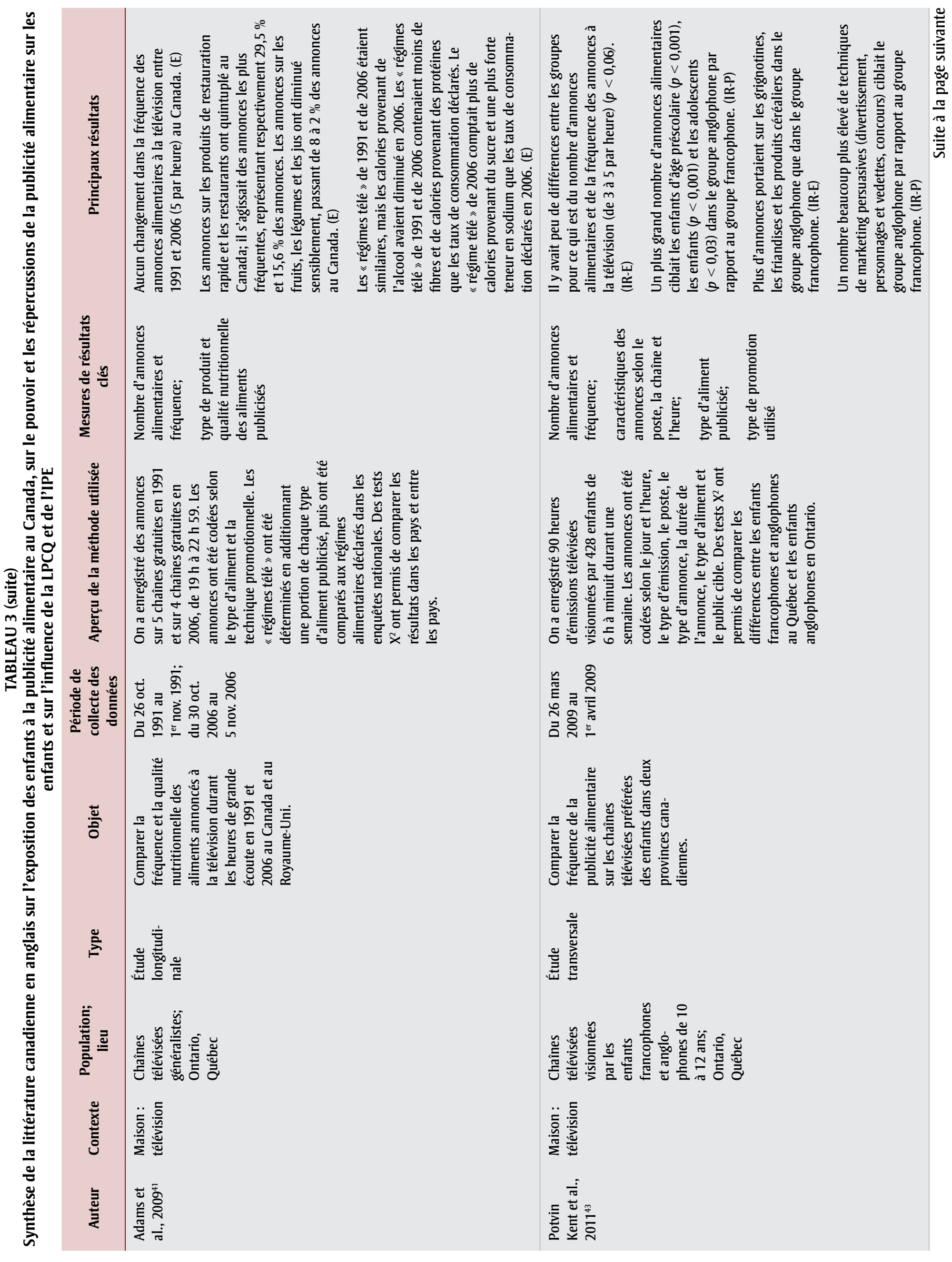




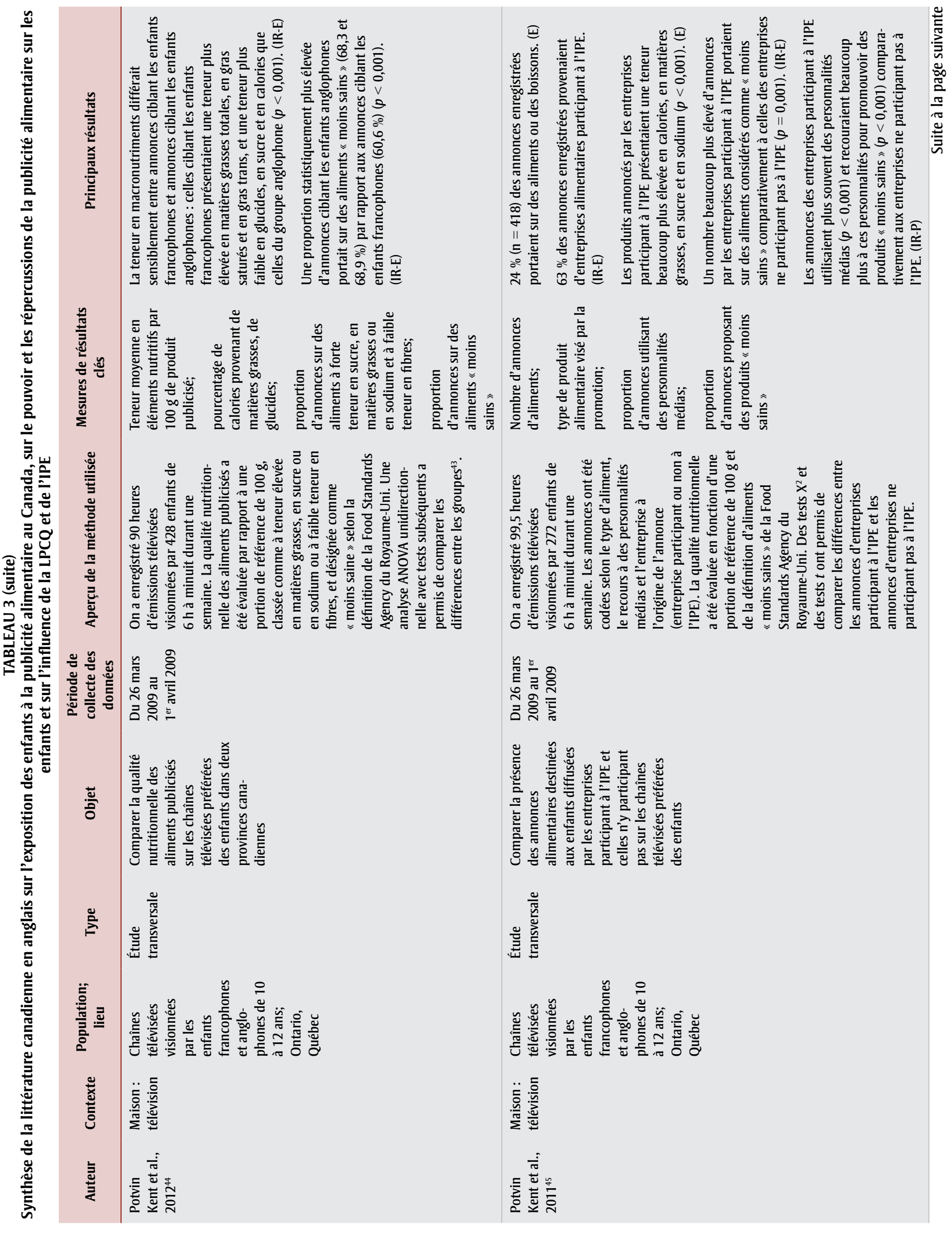




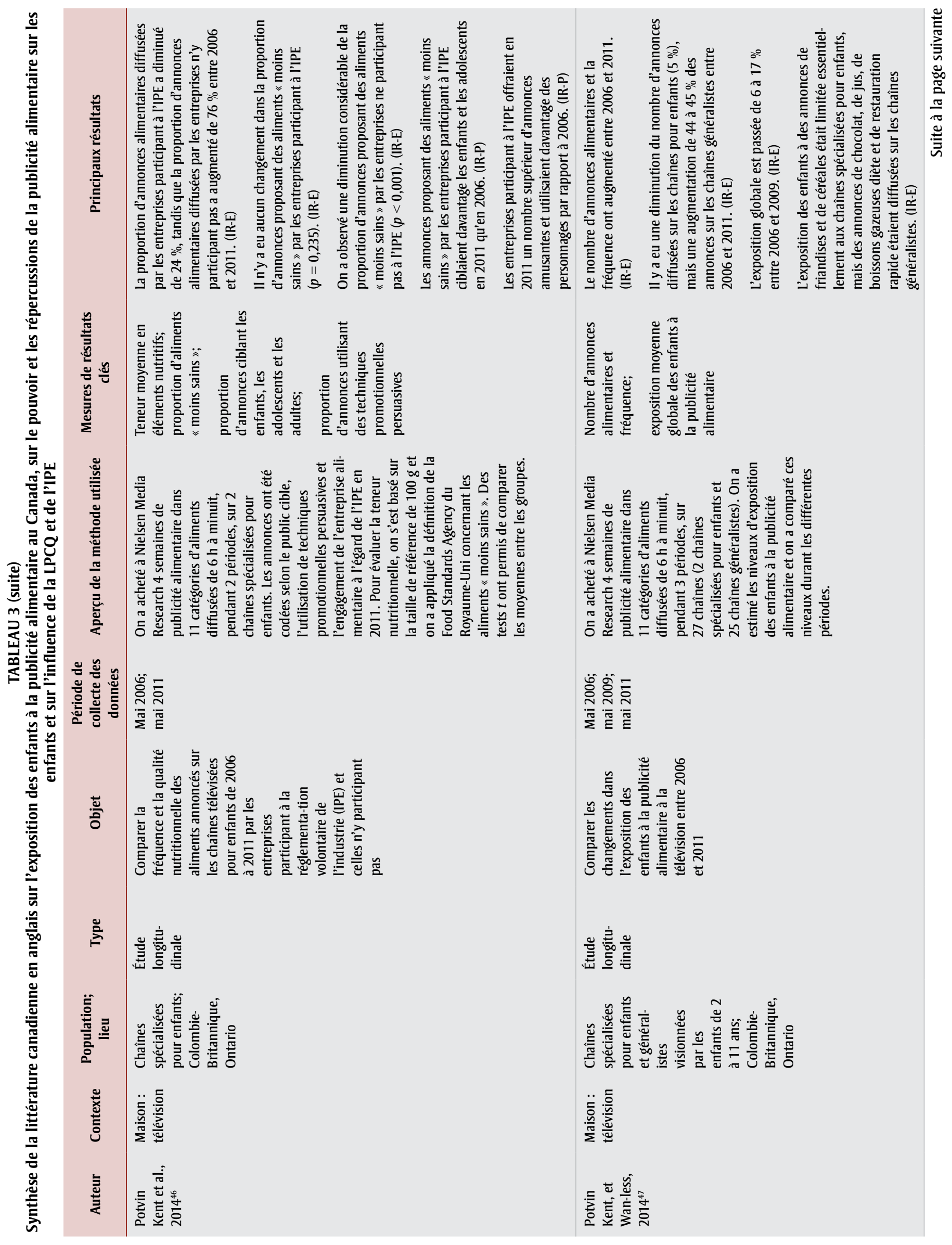




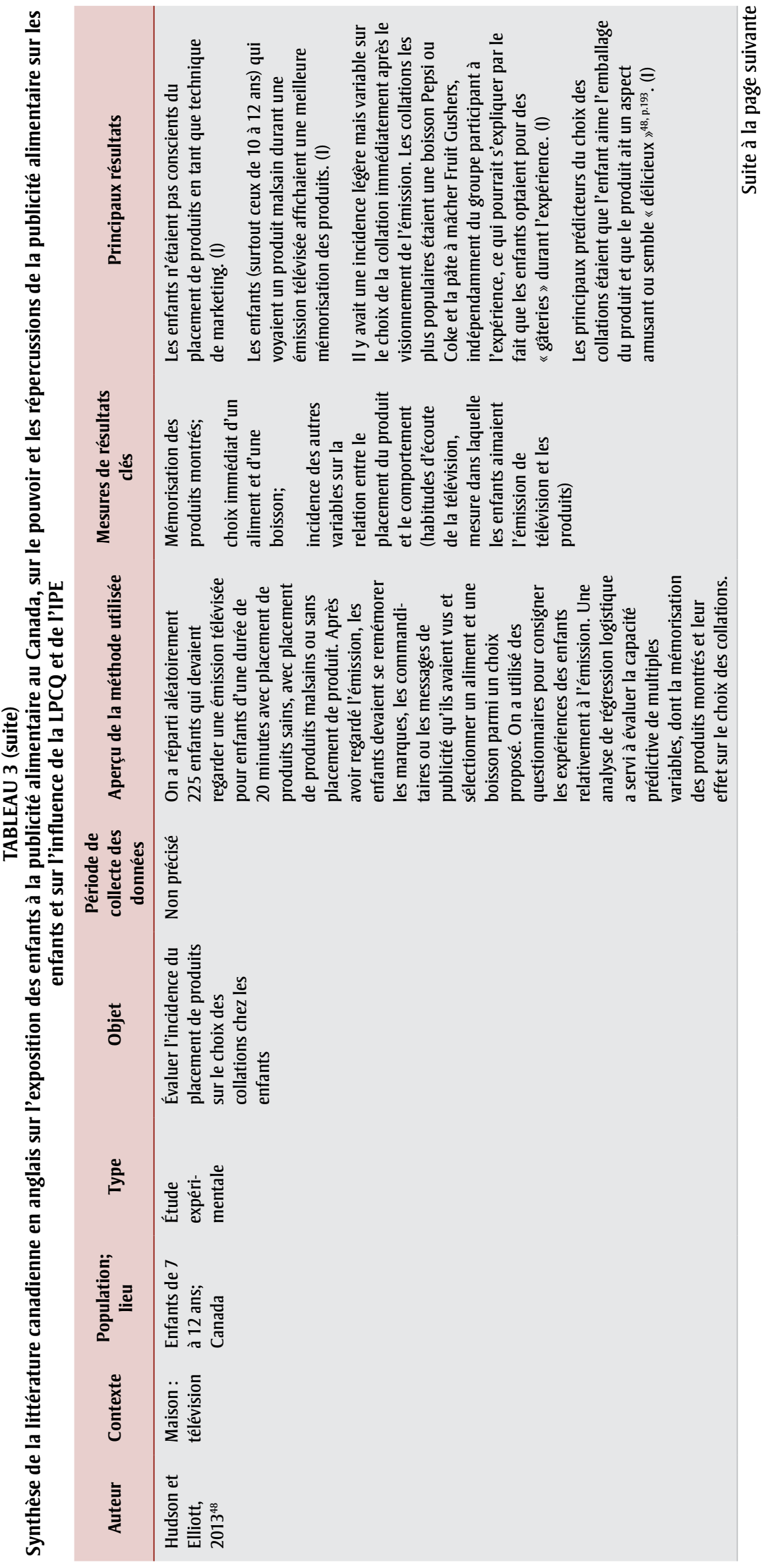

de la publicité destinée aux enfants sur les sites Internet d'entreprises alimentaires ${ }^{50,51}$. Ces études n'ont pas évalué les nombreuses nouvelles techniques de cybermarketing qui servent à cibler les enfants, notamment le marketing viral (le boucheà-oreille des consommateurs sur Internet), les réseaux sociaux et le marketing direct par courriel $l^{4}$. L'auteure n'a trouvé aucune étude portant sur ces techniques au Canada. Des études en provenance d'autres pays pourraient fournir de l'information, étant donné que les Canadiens sont en mesure d'accéder aux sites Internet étrangers. Cependant, ces sites n'étaient pas visés par notre examen. Les deux études trouvées visaient à définir les caractéristiques puissantes des sites Internet d'entreprises alimentaires et non à mesurer l'exposition (p. ex. la proportion de sites Internet contenant de la publicité alimentaire visités par des enfants). Par conséquent, les données probantes disponibles ne permettent pas de caractériser l'exposition des enfants à la publicité alimentaire sur Internet ni les répercussions de la réglementation sur le degré d'exposition.

\section{Pouvoir de la publicité alimentaire à la maison : Internet}

En 2010, Potvin Kent et al. ${ }^{51}$ ont analysé des sites Internet associés à des boissons ou à des aliments annoncés sur des chaînes télévisées regardées par les enfants de 10 à 12 ans, dans le but d'évaluer les répercussions de la LPCQ et de l'IPE. Environ le tiers des 148 sites Internet examinés étaient destinés aux enfants, c'est-à-dire " qu'ils présentaient des caractéristiques de marketing orientées vers les enfants, comme des personnages porte-parole, des dessins humoristiques, des concours, des activités ou des jeux pour les enfants et qu'ils utilisaient un vocabulaire simple, facilement compris des enfants. $»^{51, p .} 801$ [traduction]. Dans une évaluation portant uniquement sur les sites Internet d'entreprises ayant adhéré à l'IPE, $83 \%$ des sites contenaient de la publicité destinée aux enfants de moins de 12 ans $^{50}$.

Plusieurs techniques exhortaient les enfants à répondre à la publicité alimentaire sur les sites Internet des entreprises ayant adhéré à $\mathrm{l}^{\prime} \mathrm{IPE}^{50}$ :

- l'adhésion comme membre, des mesures incitatives et des super bannières pour utiliser de façon répétée et prolongée les médias en ligne; 


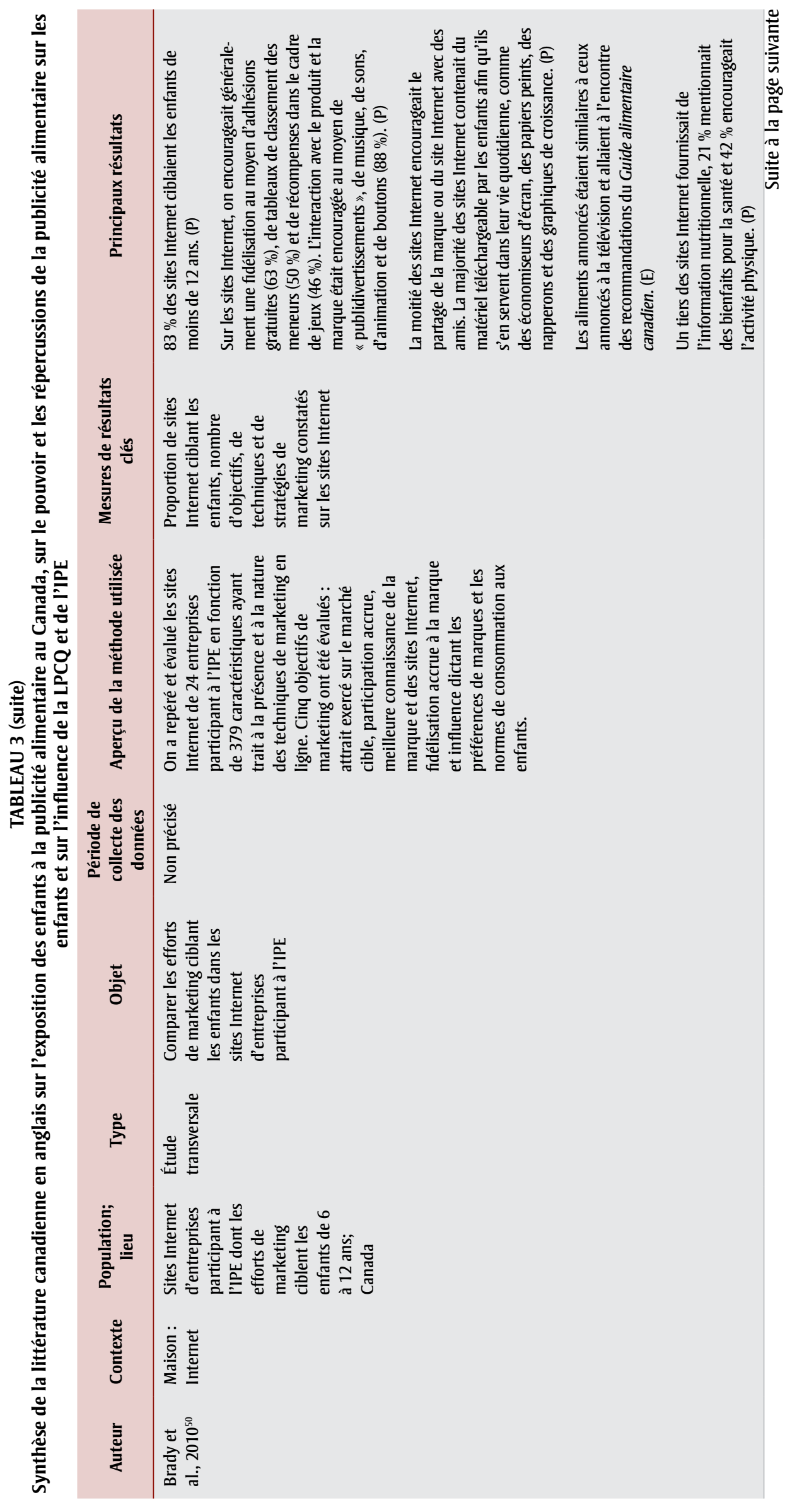

- des publidivertissements, de la musique, de l'animation et des boutons électroniques pour interagir avec le produit ou la marque;

- des techniques de bouche-à-oreille électronique pour communiquer de l'information sur une marque ou un site Internet; et

- des éléments téléchargeables (fonds d'écran, graphiques de croissance, listes d'achats, jeux de société) destinés à intégrer une marque à la vie quotidienne des enfants.

Influence de la réglementation sur le pouvoir Aucune différence statistique n'a été trouvée en matière de pouvoir de la publicité alimentaire (p. ex. enfants ciblés ou non, type ou fréquence des techniques promotionnelles utilisées) entre les sites Internet francophones et les sites Internet anglophones, ni entre les sites Internet des entreprises ayant adhéré à l'IPE et les sites Internet des autres entreprises en $2010^{51}$.

\section{Exposition à la publicité alimentaire dans les écoles}

Les données probantes sur la publicité dans les écoles sont insuffisantes : une seule étude a été menée sur le sujet en dix ans, celle de Velazquez et al. ${ }^{52}$, qui ont examiné l'étendue de la promotion alimentaire commerciale et non commerciale (faite par l'école ou les étudiants) dans un échantillon représentatif de 23 écoles publiques de Vancouver au cours de l'année scolaire 2012-2013. En utilisant l'observation, ils ont constaté que $87 \%$ des écoles faisaient de la promotion alimentaire, avec un nombre médian d'annonces promotionnelles de 17 (intervalle de variation : 0 à 57), et il y avait davantage de publicité dans les écoles secondaires que dans les écoles primaires $^{52}$.

Velazquez et al. $^{52}$ ont utilisé les lignes directrices de la Colombie-Britannique sur la nutrition en milieu scolaire ${ }^{21}$ pour évaluer le caractère sain des boissons et des aliments annoncés : plus de la moitié des écoles faisaient la promotion de boissons et d'aliments interdits par les lignes directrices provinciales ${ }^{52}$ et près du quart des annonces concernaient des produits " à choisir le moins souvent " ou " non recommandés $»^{52}$. En revanche, $80 \%$ des écoles faisaient aussi la promotion de produits "à choisir le plus souvent ", ce 


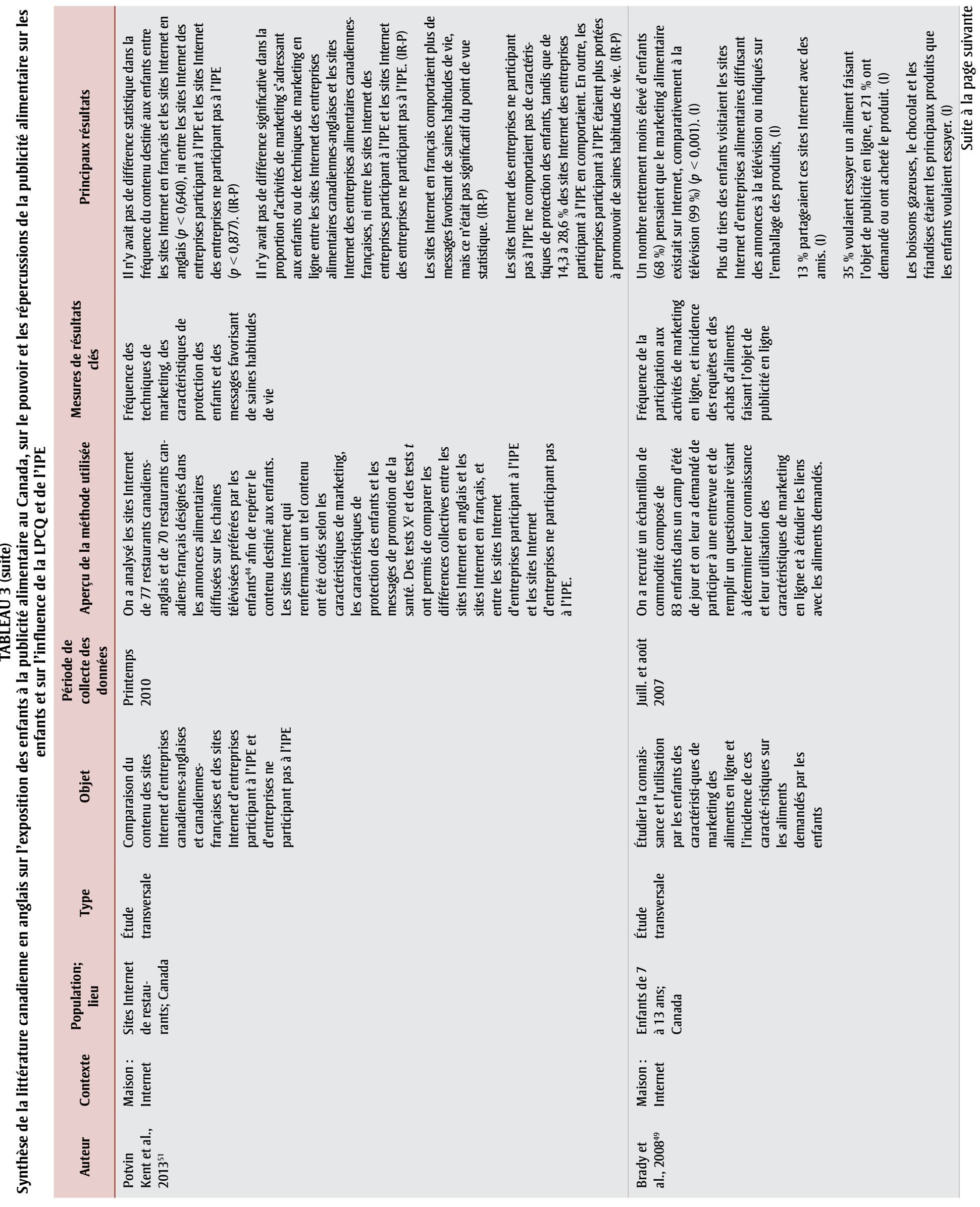




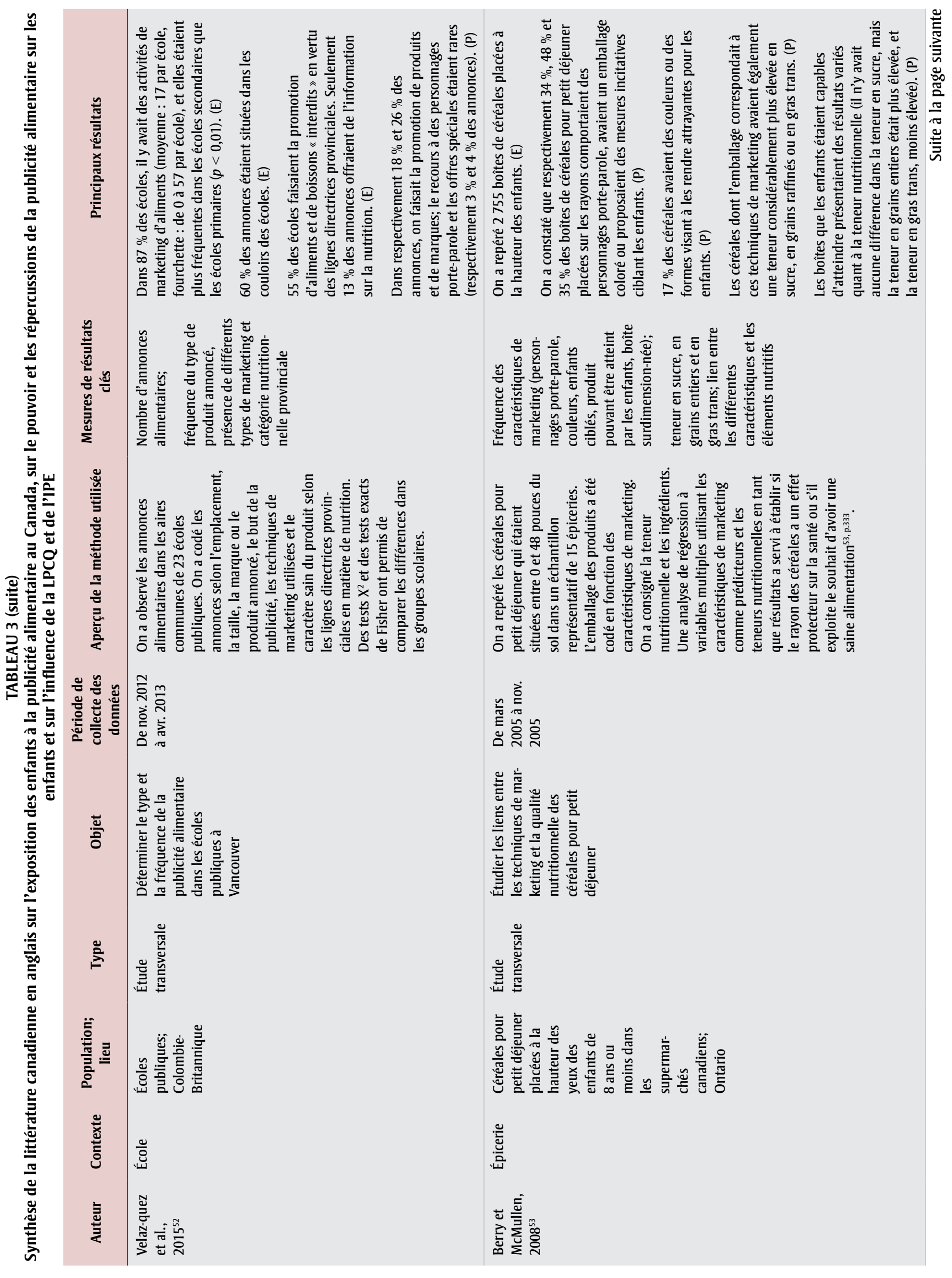




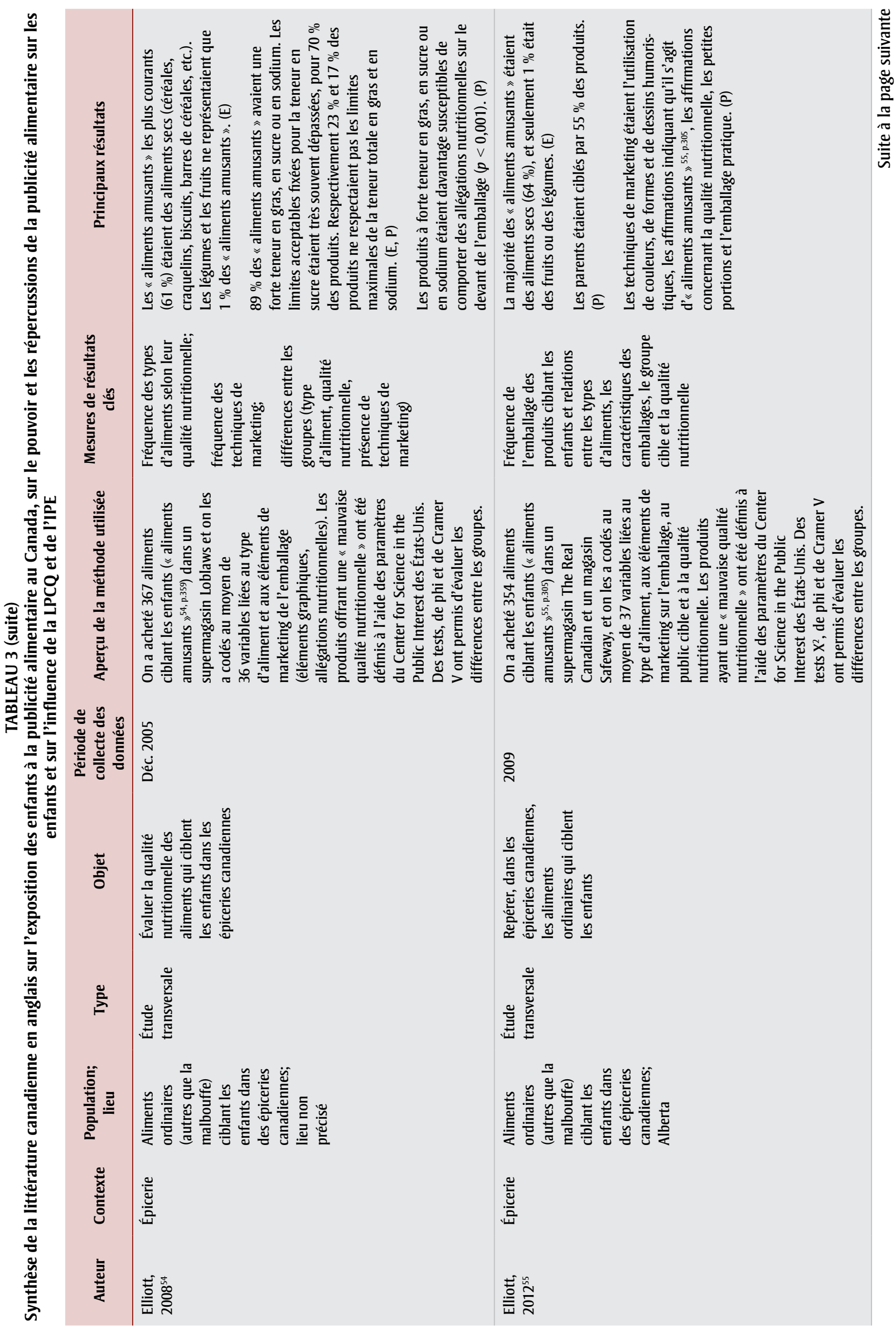




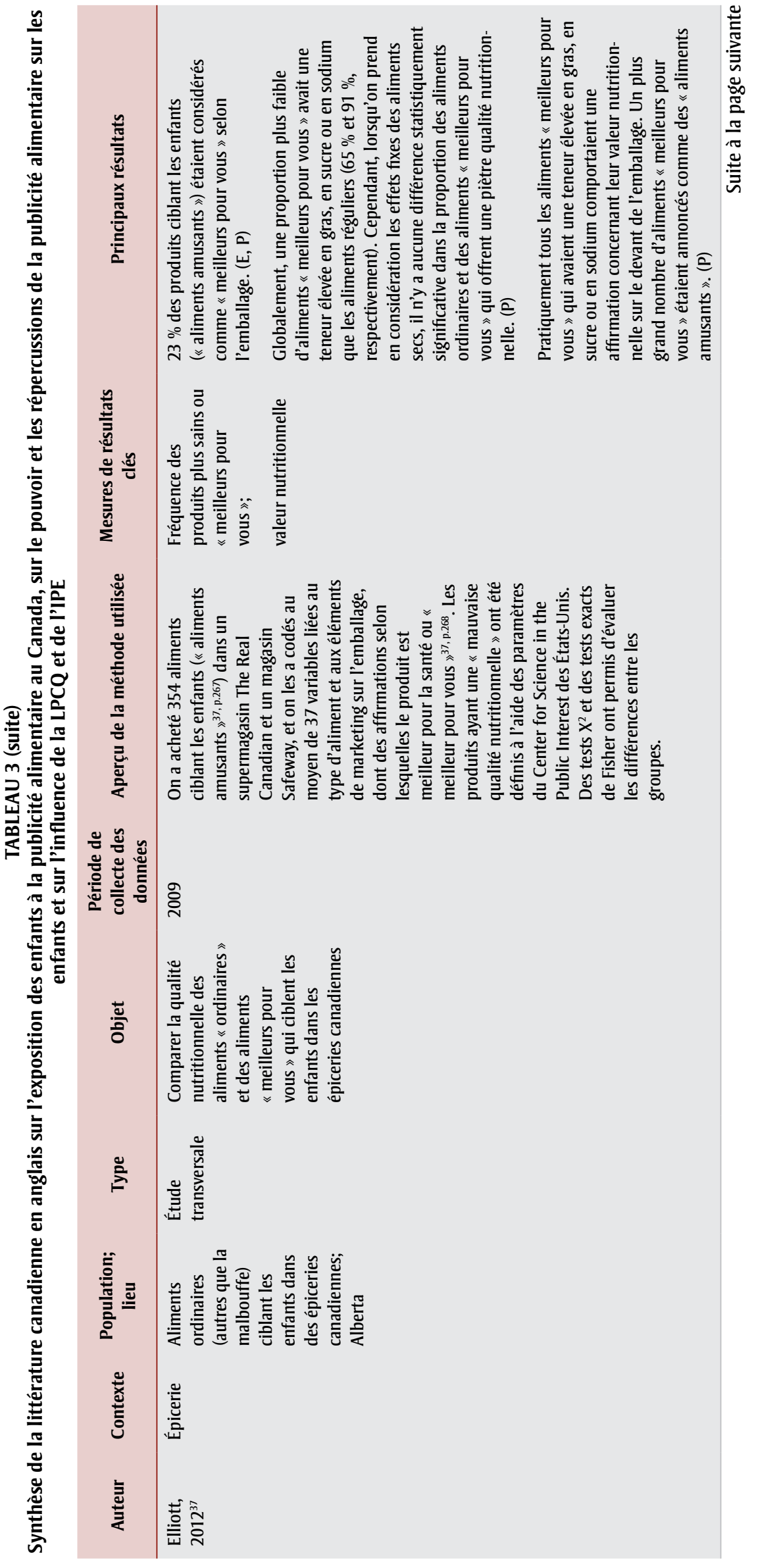

qui représentait $45 \%$ de l'ensemble des annonces promotionnelles.

Influence de la réglementation sur l'exposition Aucune étude n'a évalué les répercussions de la LPCQ ou de l'IPE sur l'exposition à la publicité alimentaire dans les écoles. Les pourcentages de publicité alimentaire les plus faibles observés dans les écoles primaires par Velazquez et al. ${ }^{52}$, dans un contexte régi en partie par l'IPE, témoignent peut-être de l'influence de l'IPE. Toutefois, ils s'expliquent probablement plutôt par le fait que les écoles secondaires offrent un plus grand nombre de services alimentaires (distributrices automatiques et comptoirs de vente d'aliments) que les écoles primaires $^{52}$ et font donc davantage de promotion alimentaire.

\section{Pouvoir de la publicité alimentaire dans les écoles}

Velazquez et al. ${ }^{52}$ ont constaté que les annonces alimentaires observées dans les écoles portaient souvent sur des marques ou des produits précis et qu'elles utilisaient rarement des personnages animés, des célébrités ou des produits en prime. Le rare recours à ces techniques puissantes pourrait provenir du fait que la moitié des annonces enregistrées n'étaient pas de nature commerciale et qu'elles avaient été créées par les étudiants ou l'école ${ }^{52}$.

\section{Influence de la réglementation sur le pouvoir Non documentée.}

\section{Exposition à la publicité alimentaire dans les supermarchés}

Deux études ont fourni des proportions de produits ciblant les enfants au moyen de l'emballage des produits. Dans 15 épiceries vérifiées au hasard en Ontario, Berry et McMullen ont observé 2755 boîtes de céréales placées à la hauteur des enfants (soit à 48 pouces maximum du sol, ce qui tient compte du niveau des yeux d'un enfant assis dans un chariot, debout ou qui marche) ${ }^{53}$. Jusqu'à la moitié du rayonnage consacré aux céréales pour petit déjeuner à la hauteur des enfants contenait des boîtes de céréales présentant au moins l'une des caractéristiques destinées aux enfants (décrites dans la section " Pouvoir de la publicité alimentaire dans les supermarchés » de cet article). Dans la base de données du Food Label Information Program de l'Université de Toronto, qui contient des données sur plus de 10000 produits alimentaires emballés recueillies en 2010 et 


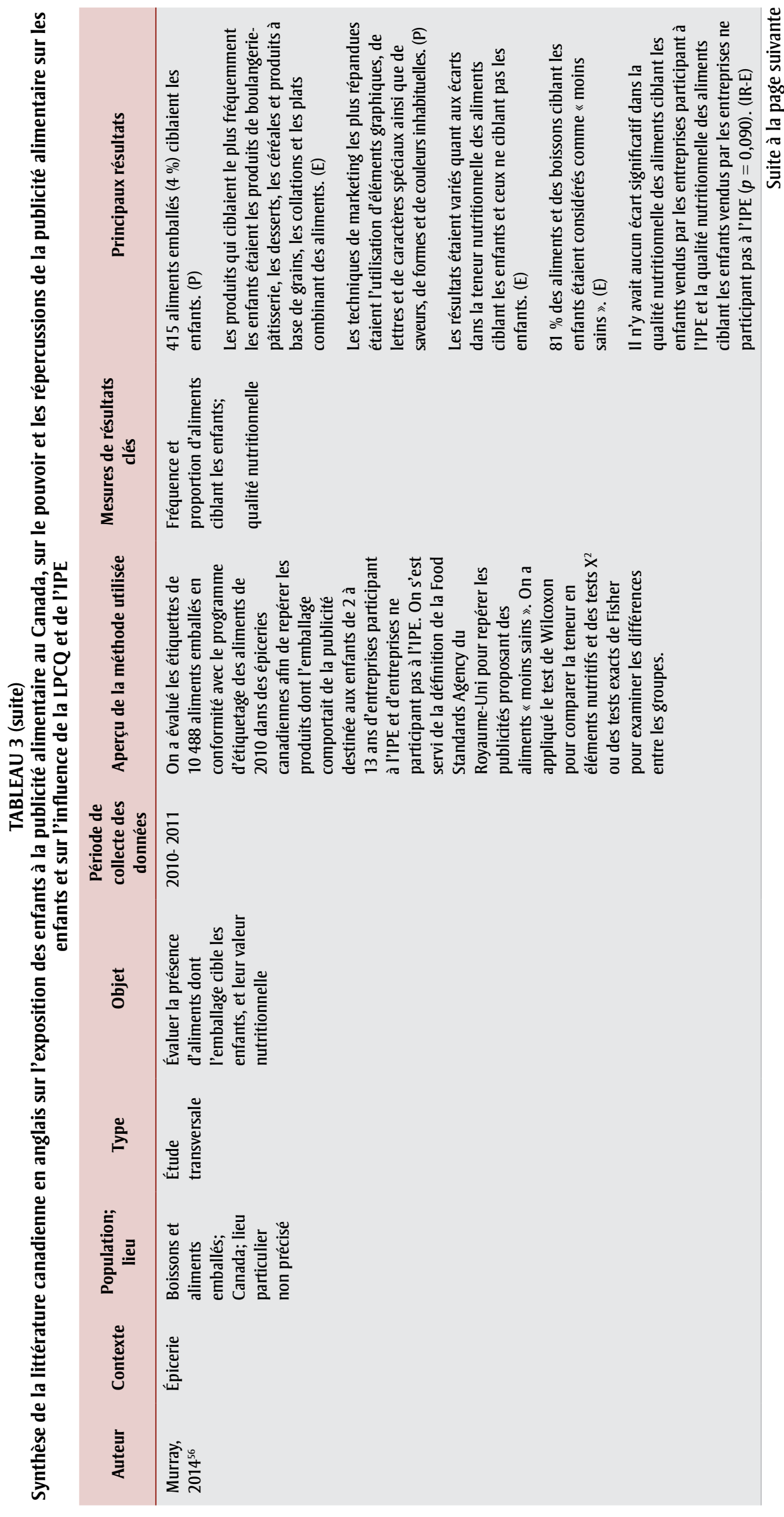

2011, Murray a découvert que 415 produits (4\%) ciblaient les enfants, c'est-à-dire qu'ils montraient des enfants qui s'amusent ou qui jouent, ou qu'ils utilisaient des dessins humoristiques ou des polices enfantines $^{56}$. Une autre étude ${ }^{55}$ a sélectionné les produits qui ne ciblaient que les enfants, sans établir de dénominateur total pour les produits : dans deux supermarchés en Alberta, Elliott a relevé plus de 350 aliments quotidiens (autres que de la malbouffe) ciblant les enfants, c'est-à-dire conçus pour les enfants ou présentant des dessins humoristiques, du marchandisage de produits associés, des formes, des couleurs ou des goûts inhabituels, ou encore des jeux sur l'emballage ${ }^{55}$. Les estimations de l'exposition dans ces trois études ne sont pas complètes : l'exposition réelle pourrait être sous-estimée, car aucune de ces études ne s'est penchée sur la publicité alimentaire près des caisses, dans des étalages à part ou liée à d'autres caractéristiques des magasins.

Dans l'ensemble, la plupart des aliments faisant l'objet d'une publicité destinée aux enfants dans les supermarchés avaient une teneur élevée en sucre, en gras ou en sodium $^{54}$ ou une faible teneur en éléments nutritifs souhaitables ${ }^{56}$. Presque le quart des aliments faisant l'objet d'une publicité destinée aux enfants portaient des étiquettes " meilleur pour vous » utilisant la définition de l'IPE, mais les deux tiers des aliments " meilleurs pour vous » présentaient tout de même une teneur élevée en sucre, en gras ou en sodium ${ }^{37}$. Une proportion sensiblement plus importante de catégories d'aliments (collations, boissons, céréales, craquelins, pouding et mets composés qui ne sont pas mesurables avec une tasse, comme la pizza) pouvaient davantage être considérées comme " moins sains » d'après le système axé sur le profil nutritionnel du Royaume-Uni lorsqu'ils étaient commercialisés pour les enfants que lorsqu'ils ne l'étaient pas ${ }^{56}$. Elliott ${ }^{55}$ et Murray $^{56}$ ont tous deux constaté que $1 \%$ ou moins des aliments commercialisés pour les enfants était constitué de fruits ou de légumes.

\section{Influence de la réglementation sur l'exposition.} Ni la LPCQ ni l'IPE ne s'appliquent explicitement à l'emballage des produits. Il n'existe aucune recherche portant sur les répercussions de la LPCQ sur l'emballage des produits. Les répercussions de l'IPE sur l'exposition générale à l'emballage des produits qui cible les enfants ne sont pas 


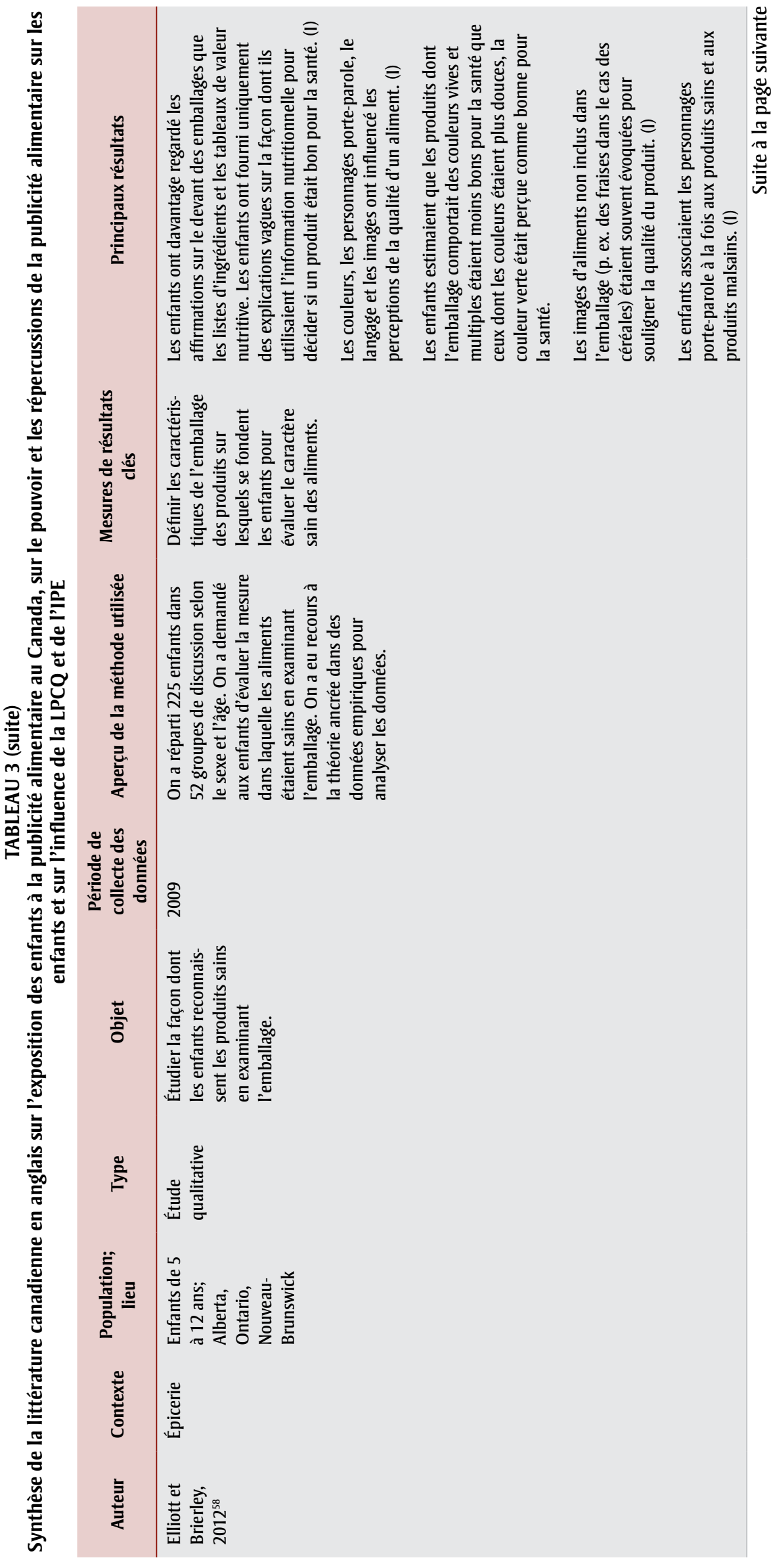

documentées. Toutefois, Murray a montré que l'IPE n'avait aucune répercussion sur la qualité nutritionnelle des aliments commercialisés pour les enfants au moyen de l'emballage des produits ${ }^{56}$.

\section{Pouvoir de la publicité alimentaire dans les supermarchés}

La plupart des produits d'épicerie examinés par Elliott présentaient un emballage comportant des caractéristiques " amusantes ", notamment des dessins humoristiques et des polices de type bande dessinée ${ }^{54}$. Murray a constaté que les saveurs, les formes et les couleurs inhabituelles, les personnages et les graphiques ou le lettrage étaient les techniques de marketing les plus couramment utilisées pour les produits ciblant les enfants ${ }^{56}$. Dans une analyse des boîtes de céréales pour petit déjeuner, $48 \%$ portaient des couleurs à l'intention des enfants, $35 \%$ offraient des mesures incitatives ou des produits en prime et $34 \%$ avaient des personnages porte-parole ${ }^{53}$.

De façon similaire aux résultats de recherche sur les annonces alimentaires télévisées, les techniques de marketing puissantes sur l'emballage des produits étaient associées à des aliments de mauvaise qualité ${ }^{33,54}$. En particulier, les céréales pour petit déjeuner étaient davantage susceptibles d'avoir une teneur élevée en sucre si leur emballage ciblait les enfants ${ }^{53}$. De même, plus des deux tiers des produits à haute teneur en sucre (n'étant pas de la malbouffe) comportaient des allégations de qualités nutritionnelles, comparativement à seulement la moitié des produits " plus sains $»^{54}$. Berry et McMullen ont avancé que le paysage marketing dans l'allée des céréales au Canada « exploite la santé »"53.p.334, c'est-à-dire qu'il utilise des techniques de marketing destinées aux enfants pour promouvoir des produits moins sains, ce qui incite à les consommer.

\section{Influence de la réglementation sur le pouvoir Non documentée.}

\section{Répercussions de la publicité alimentaire sur les enfants au Canada}

Les données établissant un lien de cause à effet entre la publicité alimentaire et les attitudes, les préférences et les comportements alimentaires des enfants sont convaincantes et ont été analysées ailleurs ${ }^{6,61,62}$. Même si elles sont limitées, les études canadiennes donnent une idée, à l'échelle 


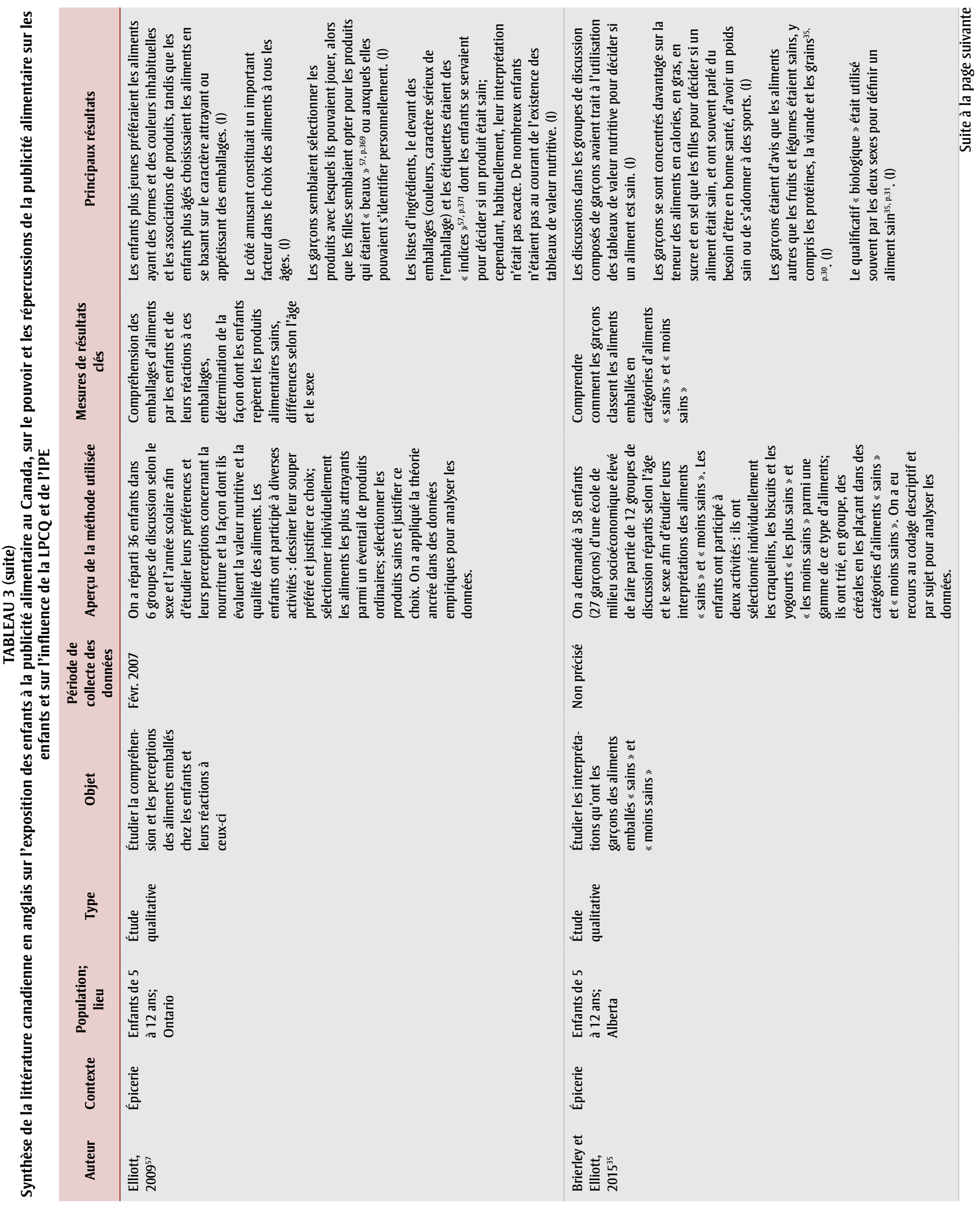




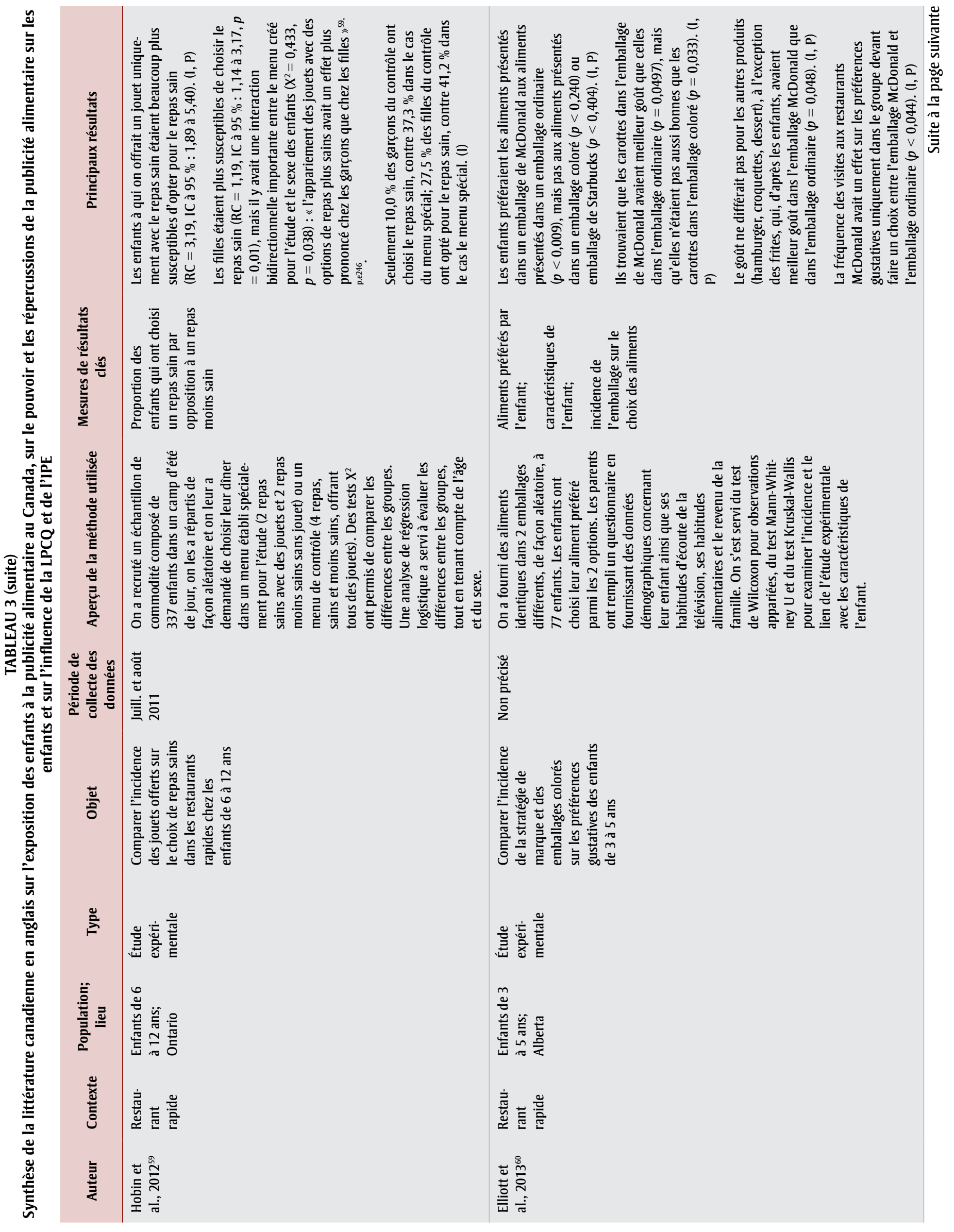




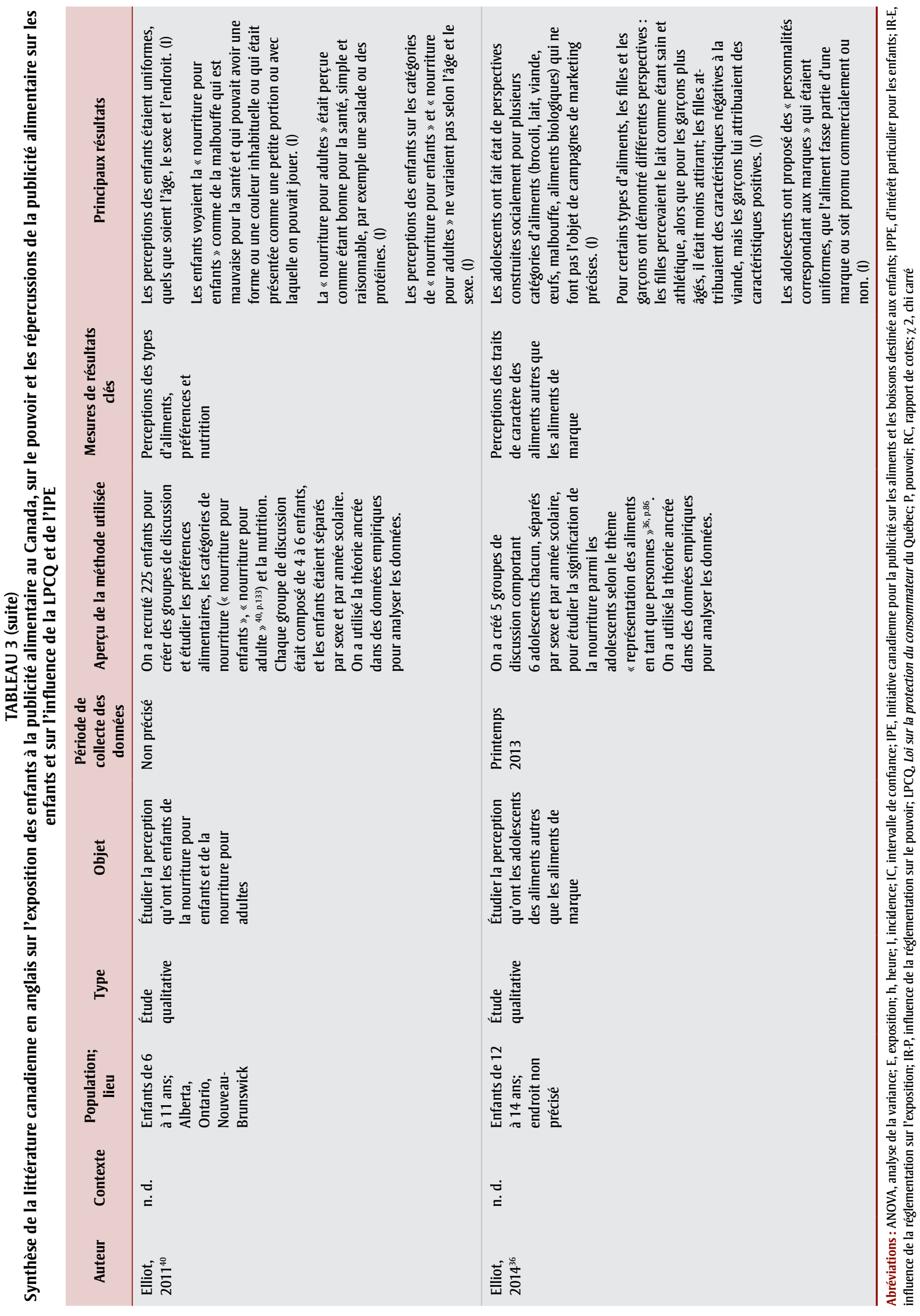


TABLEAU 4

Résumé de l'influence de la réglementation actuelle au Canada sur l'exposition des enfants à la publicité alimentaire et sur le pouvoir qu'elle exerce sur ceux-ci, selon le contexte

\begin{tabular}{|c|c|c|c|c|c|c|}
\hline \multirow[b]{2}{*}{ Contexte } & \multicolumn{4}{|c|}{ Influence de la LPCQ } & \multicolumn{2}{|l|}{ Influence de I'IPE } \\
\hline & $\begin{array}{l}\text { Expositiona à la } \text { publicité } \\
\text { alimentaire en } \\
\text { général }\end{array}$ & $\begin{array}{l}\text { Expositiona à la } \\
\text { publicité sur des } \\
\text { aliments } \\
\text { malsains }\end{array}$ & Pouvoir $^{\mathbf{b}}$ & $\begin{array}{l}\text { Expositiona à la publicité } \\
\text { alimentaire en général }\end{array}$ & $\begin{array}{l}\text { Expositiona à la } \\
\text { publicité sur des } \\
\text { aliments } \\
\text { malsains }\end{array}$ & Pouvoir $^{b}$ \\
\hline $\begin{array}{l}\text { Maison } \\
\text { (télévision) }\end{array}$ & Aucune influence & $\begin{array}{l}\text { Influence } \\
\text { positive }\end{array}$ & $\begin{array}{l}\text { Influence } \\
\text { positive }\end{array}$ & Influence négative & Aucune influence & $\begin{array}{l}\text { Influence } \\
\text { négative }\end{array}$ \\
\hline École & - & - & - & - & - & - \\
\hline Supermarché & - & - & - & - & Aucune influence & - \\
\hline
\end{tabular}

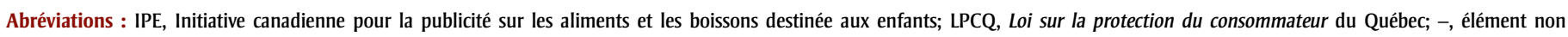
documenté.

${ }^{a}$ Le terme « exposition» est défini comme étant « la portée et la fréquence du message publicitaire » 9, p.11.

${ }^{\mathrm{b}}$ Le terme « pouvoir » est défini comme étant « la conception, l'exécution et le contenu créatifs du message publicitaire » ${ }^{9, p .11}$.

locale, de la façon dont les enfants canadiens subissent les répercussions de la publicité alimentaire. Les études expérimentales et qualitatives au Canada ont montré que le placement de produits à la télévision $^{48}$, la publicité sur Internet ${ }^{49}$, l'emballage des produits ${ }^{35,57,58,60}$ et les jouets offerts en prime ${ }^{59}$ peuvent avoir des répercussions sur les attitudes, les préférences et les comportements alimentaires des enfants canadiens.

Hudson et Elliott ${ }^{48}$ ont constaté que, même si seulement $17 \%$ des enfants (7 à 12 ans) étaient conscients du placement de produits, ceux qui avaient regardé une émission télévisée contenant un placement de produits malsains (comparativement à une émission télévisée sans placement de produit ou contenant un placement de produits sains) étaient davantage susceptibles de se rappeler les produits annoncés. Presque le quart des enfants de 7 à 13 ans ont dit avoir acheté ou demandé un aliment annoncé sur Internet (le plus souvent des boissons gazeuses, du chocolat et des friandises) ${ }^{49}$.

Des chercheurs ont organisé des groupes de discussion composés d'enfants de 5 à 12 ans pour évaluer leurs préférences, leurs perceptions et leurs interprétations des aliments emballés ${ }^{35,57,58}$. Leurs préférences étaient souvent influencées par l'emballage utilisant des thèmes amusants et plaisants sur le plan esthétique ou interactif ${ }^{57}$. Lorsqu'on leur demandait de nommer des produits sains, les enfants créaient leurs propres arguments (souvent inexacts) en fonction des couleurs $^{57}$, des allégations nutritionnelles ou biologiques ${ }^{35,57}$, des listes d'ingrédients ${ }^{35,57}$ et, parfois seulement, des tableaux de valeur nutritive ${ }^{35,57}$. Les résultats de ces groupes de discussion composés de 225 enfants de l'ensemble du Canada ont révélé que les caractéristiques liées au marketing (couleurs, mots, dessins, personnages porte-parole et allégations sur le devant de l'emballage) étaient utilisées plus souvent que la valeur nutritive et les listes d'ingrédients dans l'évaluation du caractère sain des aliments emballés ${ }^{58}$.

Elliot et al. ${ }^{60}$ se sont demandé si les préférences gustatives des enfants de 6 à 11 ans différaient selon la conception de l'emballage des aliments. D'après leur étude, les enfants préféraient les aliments présentés dans un emballage de McDonald aux aliments présentés dans un emballage neutre, mais cette préférence disparaissait lorsque les aliments présentés dans un emballage de McDonald étaient comparés à des aliments dans des emballages colorés ou dans des emballages de Starbucks. Dans l'étude d'une méthode de promotion des aliments sains, Hobin et al..$^{59}$ ont évalué les répercussions de l'offre de jouets en prime sur le choix des repas. Les enfants (6 à 12 ans) à qui on offrait des jouets en prime avec des aliments sains seulement (par rapport aux aliments sains et malsains) étaient plus de trois fois plus susceptibles de choisir le repas $\operatorname{sain}^{59}$.

Enfin, des données probantes d'études qualitatives non fondées sur le contexte ont révélé que les enfants canadiens ont des attitudes homogènes envers les aliments $^{36,40}$, ce qui laisse penser que les expositions cumulatives à la publicité alimentaire sont susceptibles d'avoir de plus grandes répercussions sur la culture alimentaire des enfants que ce que mesure une exposition simple dans le cadre d'une étude. Des groupes de discussion menés en Alberta, en Ontario et au NouveauBrunswick avec des enfants de 6 à 11 ans ont montré que les enfants font la distinction entre les aliments qui leur sont destinés et les aliments destinés aux autres ${ }^{40}$. Ils ont déclaré que les « aliments pour enfants » étaient de la malbouffe sucrée, associée à des dessins humoristiques et offerte en formes ou en couleurs amusantes, ces aliments pouvant être consommés avec les mains ou servir de jouets $^{40, p .133}$. Ces caractéristiques symboliques nommées par les enfants sont le reflet des techniques puissantes énumérées dans notre étude et utilisées par l'industrie alimentaire pour commercialiser un produit auprès des enfants. Inversement, les enfants percevaient les aliments pour adultes comme étant fades, non transformés, sains, responsables et pas pour eux ${ }^{40}$. De même, les adolescents (de 12 à 14 ans) ont personnifié les aliments de façon uniforme dans l'ensemble du Canada ${ }^{36}$. Le brocoli est « timide, impopulaire et ennuyant $»^{36, p . ~} 87$ et le lait est « athlétique $»^{36, \text { p. } 87}$ (à l'exception des garçons plus âgés). Ils ont identifié la malbouffe à une "personne qui aime faire la fête ", qui est " amusante et agréable à côtoyer $\aleph^{36, p . ~} 87$. Il est possible que les attitudes alimentaires des enfants aient été construites socialement en fonction de la publicité alimentaire commerciale ou de 
son absence, ce qui pourrait expliquer en partie pourquoi l'alimentation des enfants n'est pas en harmonie avec les recommandations du Guide alimentaire canadien.

\section{Analyse}

Notre étude de portée témoigne de l'exposition multiple des enfants à la publicité alimentaire dans différents contextes : à la maison, à l'école et dans les supermarchés. À l'exception de la télévision et de l'emballage des produits, les données probantes sont limitées. Les restaurants rapides constituent un contexte où l'on pourrait s'attendre à trouver de la publicité alimentaire, mais seules les répercussions des techniques promotionnelles utilisées dans ces restaurants ${ }^{59,60}$ ont été étudiées au Canada. Les travaux de recherche portant sur d'autres pays ont fait état de publicité alimentaire dans d'autres contextes (restaurants $^{63}$, complexes sportifs ${ }^{64}$ et à l'extérieur ${ }^{65}$ ), ce qui fait que notre examen sous-estime probablement l'exposition des enfants canadiens. Les aliments à teneur élevée en calories, en gras, en sucre et en sodium étaient généralement commercialisés dans tous les contextes, ce qui correspond aux résultats d'autres recherches ${ }^{6}$. Les enfants étaient souvent ciblés à l'aide de techniques promotionnelles puissantes, nombreuses et variées, qui se chevauchaient entre contextes. Les spécialistes du marketing alimentaire disposent d'un arsenal important d'outils de marketing.

À l'exception des influences positives limitées de la réglementation officielle au Québec sur les publicités alimentaires télévisées, les données probantes actuelles suggèrent que la réglementation officielle et l'autoréglementation au Canada n'ont amélioré ni l'exposition des enfants ni le pouvoir de la publicité alimentaire. Il faut cependant approfondir les recherches pour comprendre l'incidence de la réglementation dans l'ensemble des contextes. Dhar et Baylis ont estimé que la LPCQ avait une incidence positive sur la santé de la population, en réduisant la consommation hebdomadaire d'aliments prêt-à-manger à la maison chez les francophones, mais pas chez les ménages anglophones avec enfants résidant au Québec, puisque ces derniers pourraient regarder des publicités alimentaires ne provenant pas du Québec, et n'étant donc pas assujetties à la $\mathrm{LPCQ}^{66}$. Bien que l'influence de la réglementation dans les écoles n'ait pas été mesurée, un sondage mené en 2004 auprès de toutes les écoles publiques canadiennes a révélé que la fréquence de la publicité commerciale (aliments et autres) était plus faible au Québec que dans le reste du Canada ${ }^{67}$. La réglementation officielle au Québec, une approche fondée sur les droits de la santé des enfants ${ }^{4}$, pourrait avoir une meilleure influence sur les conditions et le contexte dans lesquels vivent les enfants que l'autoréglementation de l'industrie.

La synthèse des données probantes présentée ici montre que les attitudes, les préférences et les comportements alimentaires des enfants canadiens subissent les répercussions de l'exposition à la publicité alimentaire dans tous les contextes. Toutefois, l'uniformité des attitudes alimentaires chez les enfants canadiens est peut-être encore plus importante, ce qui donne à penser que l'exposition à la publicité alimentaire au fil du temps et dans l'ensemble des contextes a des répercussions collectives et globales. Étant donné que les enfants sont de plus en plus immergés dans la publicité en grandissant et que les techniques promotionnelles et les réseaux s'intègrent et se chevauchent de plus en plus souvent ${ }^{68}$, il est raisonnable de penser que l'exposition à la publicité sur les aliments malsains a des répercussions cumulatives plus grandes ${ }^{61}$ que lorsque qu'elle est étudiée séparément par type de promotion.

L'ensemble des données probantes fournies dans notre étude de portée doit être restitué dans le cadre du quotidien d'un enfant canadien moyen, qui regarde la télévision deux ou trois heures par jour ${ }^{69}$, utilise l'ordinateur ou joue à des jeux vidéo une ou deux heures par jour ${ }^{69}$, est assis à l'école cinq ou six heures par jour ${ }^{70}$ et dont la famille fait l'épicerie presque tous les deux jours $^{71}$. Sous cet angle, il devient encore plus évident que les enfants au Canada (à l'exception de certains au Québec) sont susceptibles d'être exposés à un volume incroyable de publicité alimentaire puissante. En outre, les contextes de publicité alimentaire sélectionnés par l'auteure dans cette étude constituent des situations courantes dans lesquelles les enfants consomment des aliments, en achètent ou apprennent à les connaître.

L'étude des écoles de Vancouver invite à penser que l'exposition des enfants à la publicité sur les aliments malsains est moins fréquente et que la publicité est moins puissante dans les écoles que dans d'autres contextes, car seul le quart des aliments annoncés étaient malsains, et les techniques promotionnelles puissantes étaient rares $^{52}$. Cette constatation mérite d'être soulignée, car cela tend à signifier que les politiques axées sur le contexte comme la politique obligatoire de Colombie-Britannique sur l'alimentation en milieu scolaire qui contient des recommandations relatives à la publicité alimentaire $^{21,22}$ - sont plus globales et efficaces que les règlements axés sur la promotion traditionnelle. Cela pourrait ne pas concerner la vaste commercialisation des aliments dans les écoles publiques canadiennes déjà mentionnée ${ }^{67}$, notamment les accords d'exclusivité avec Coca-Cola et Pepsi, les programmes incitatifs (Étiquettes pour l'éducation de Campbell) et le matériel pédagogique parrainé (programme "Book It! » de Pizza Hut et programme "Smart Cookie » de M. Christie). Malheureusement, le faible nombre de recherches dans ce domaine empêche de tirer des conclusions sur la situation de la commercialisation dans les écoles, en particulier parce que la variabilité des politiques alimentaires en milieu scolaire contribue probablement à des environnements de publicité alimentaire différents selon les écoles au Canada.

Les experts recommandent des règlements officiels rigoureux et globaux, une surveillance indépendante et des pénalités en cas de non-respect pour réduire efficacement l'exposition des enfants à une publicité sur les aliments malsains puissante $e^{72,73}$. Ceux qui planifient les interventions doivent tenir compte de la façon dont l'exposition multiple à la publicité alimentaire interagit et contribue à construire socialement les attitudes et les comportements alimentaires des enfants dans leur quotidien. La tendance des règlements à être axés sur les aspects promotionnels de la publicité alimentaire ${ }^{74}$ sans tenir compte des contextes dans lesquels les enfants consomment des aliments, en achètent ou apprennent à les connaître peut augmenter le risque pour les politiques de ne pas intercepter adéquatement les plans des spécialistes du marketing en vue d'atteindre les enfants. L'importance des contextes en tant que composante de la réglementation canadienne sur la publicité alimentaire en préparation ${ }^{17}$ prend forme si la réglementation tient compte des contextes non seulement en tant que réseaux promotionnels commerciaux, mais aussi en tant que places où ont lieu des comportements et où sont achetés des produits et services ${ }^{5}$ - où les enfants consomment et achètent des aliments, ou s'informent à leur sujet. 


\section{Répercussions pour les politiques et la recherche}

Une approche exhaustive visant à restreindre la publicité sur les aliments malsains destinée aux enfants, c'est-à-dire une approche qui tienne compte du produit, de la promotion, du prix et de la distribution, pourrait nécessiter des mesures à la fois de la part des décideurs, de l'industrie et des collectivités.

Aux États-Unis, Palaskhappa et al. ont constaté que la diminution de l'obésité infantile était associée aux lois rigoureuses régissant la vente d'aliments malsains (rapport de cotes $=0,68$; intervalle de confiance [IC] à $95 \%: 0,48$ à 0,96 ) et la publicité alimentaire dans les écoles (rapport de cotes $=0,63$; IC à $95 \%: 0,46$ à $0,86)$ dans certains États, comparativement aux États sans législation ${ }^{75}$. En outre, les États disposant de plusieurs lois rigoureuses sur l'alimentation dans les écoles (deux ou plus) comparativement aux États sans législation avaient réduit le risque d'obésité dans les écoles primaires et de surplus de poids dans les écoles intermédiaires $^{75}$. Le succès de ce type de réglementation démontre que la politique gouvernementale qui régit l'industrie alimentaire, si elle donne suite aux recommandations issues de la recherche ${ }^{73}$, peut se combiner aux initiatives des milieux locaux afin d'interdire la publicité sur les aliments malsains dans les endroits où les enfants vivent, apprennent et jouent, en particulier les écoles et les installations récréatives. Les endroits où nous consommons et achetons des aliments et nous nous informons sur ceux-ci sont des lieux essentiels d'intervention pour la promotion de la santé, tout comme ils constituent des cibles essentielles pour l'industrie alimentaire.

L'objectif des restrictions sur la publicité alimentaire devrait être d'améliorer le quotidien des enfants plutôt que de simplement limiter les réseaux commerciaux utilisés pour les atteindre. Le fait de se concentrer uniquement sur les aspects promotionnels de la publicité alimentaire semble permettre aux spécialistes du marketing de conserver leur accès aux enfants simplement en passant d'une technique de marketing à une autre. L'augmentation des nouvelles techniques de marketing dans les médias et la diminution de la publicité télévisée observées aux États-Unis après l'introduction de l'autoréglementation industrielle ${ }^{68}$ pourraient être la preuve de ce type de conséquence. Les secteurs qui diffusent de la publicité sur les aliments (écoles, médias, détaillants, organisations sportives, etc.) sont des acteurs clés dans le soutien aux restrictions sur la publicité alimentaire?

En utilisant le code de l'industrie de radiodiffusion comme exemple de mesure fondée sur le secteur ${ }^{12}$, les organisations et les collectivités peuvent assumer le rôle de chef de file dans les interventions axées sur le contexte en élaborant leurs propres politiques de parrainage ou de marketing qui tiennent compte de la promotion, de la distribution et du prix des boissons et des aliments malsains. La promotion de la santé fondée sur le contexte aide à passer d'une approche des facteurs de risque fondée sur l'individu à une approche qui reconnaît la complexité des facteurs individuels et environnementaux interdépendants qui ont une influence sur la santé ${ }^{14}$. Les approches systémiques globales, une caractéristique des interventions axées sur le contexte, combinées avec des mesures prises par le gouvernement, l'industrie et les collectivités, pourraient avoir une incidence plus vaste sur la culture que les approches simplistes traditionnelles qui envisagent les problèmes sous un angle purement linéaire, avec des relations uniquement de cause et effet ${ }^{14}$. Par exemple, les politiques alimentaires des écoles, qui peuvent cibler plusieurs aspects de la publicité (voir tableau 1), peuvent être étoffées afin d'aborder globalement les « $4 \mathrm{P}$ ». En plus de la réglementation sur la publicité proposée, les décideurs pourraient également envisager d'adopter des interventions supplémentaires de soutien ciblant plus largement les caractéristiques des « $4 \mathrm{P}$ » des spécialistes du marketing, comme une disponibilité en produits liée à un changement de composition effectué par l'industrie ou un prix des aliments lié à des taxes et des subventions, dans le cadre d'une intervention globale en vue de réduire les répercussions de la publicité alimentaire. Une stratégie politique axée sur les « $4 \mathrm{P}$ » pourrait aider à recadrer la publicité sur les aliments malsains dans les situations où il n'est pas pertinent ou possible d'introduire une politique fondée sur le contexte, comme dans le secteur des affaires.

Il est nécessaire d'intensifier les recherches pour examiner de manière approfondie, en tenant compte de l'influence des "4P », l'exposition des enfants à la publicité alimentaire, le pouvoir qu'elle exerce sur eux et les répercussions qu'elle a dans leur quotidien. Plus précisément, davantage de recherches sont nécessaires sur la façon dont les divers milieux, en particulier les écoles, les centres de loisirs, les garderies et les détaillants, pourraient être ciblés par une nouvelle politique protégeant les enfants de la publicité sur les aliments malsains. Il est nécessaire également d'intensifier les recherches sur les jeunes de plus de 12 ans et sur certains sous-groupes de population (p. ex. en fonction du revenu ou de l'ethnicité) pour avoir une vue d'ensemble de la publicité alimentaire destinée aux enfants au Canada et de ses répercussions.

\section{Forces et limites}

L'approche axée sur le contexte ${ }^{18}$ adoptée dans le cadre de notre étude diverge du point de vue habituel, qui se focalise sur les médias et la promotion. Elle permet un regard neuf sur l'exposition des enfants à la publicité alimentaire ainsi que sur le pouvoir et les répercussions que celle-ci peut avoir dans leur vie. En analysant la littérature dans l'optique des « $4 \mathrm{P}$ » du marketing, notre étude fait un lien entre santé de la population et domaine des affaires. Elle offre une nouvelle perspective sur les interventions en matière de santé de la population et sur la recherche sur la publicité alimentaire destinée aux enfants.

Restreintes aux articles évalués par les pairs et à la recherche anglophone au Canada, les constatations de notre étude sont susceptibles de sous-estimer l'exposition des enfants à la publicité alimentaire au Canada et le pouvoir que celle-ci exerce sur eux. Notre stratégie de recherche, par les limites qui lui ont été fixées, peut avoir conduit à exclure des études qui mesuraient de façon superficielle la publicité alimentaire destinée aux enfants, dans le cadre d'objectifs plus larges non pertinents pour notre examen. Avec seulement 23 études (la plupart transversales) publiées en 10 ans, les évolutions de la publicité ne sont pas bien documentées. De plus, en raison de la diversité de conception des études, leur qualité n’a pas été évaluée.

\section{Conclusion}

Créer des milieux favorisant de saines habitudes alimentaires chez les enfants constitue une stratégie prioritaire de réduction de l'obésité infantile au Canada ${ }^{76}$. Toutefois, la publicité alimentaire dans les contextes dans lesquels les enfants 
consomment des aliments, en achètent ou apprennent à les connaître encourage la malbouffe " amusante " et ne favorise pas une saine alimentation. Les résultats de notre étude de portée laissent penser que la réglementation officielle et l'autoréglementation ne protègent pas adéquatement les enfants canadiens de l'exposition à une publicité sur les aliments malsains puissante. Des actions complémentaires du gouvernement, de l'industrie et des collectivités, telles une réglementation officielle forte, imposée et surveillée et des politiques alimentaires plus larges dans les écoles, pourraient être nécessaires pour aborder la nature multidimensionnelle de cette publicité sur les aliments puissante. Avec presque sept millions d'enfants de moins de 18 ans $^{77}$ au Canada et 400000 nouvelles naissances chaque année ${ }^{78}$, protéger de la publicité sur les aliments malsains les endroits où les enfants vivent, apprennent et jouent constitue l'une des stratégies nécessaires pour aider à inverser le cours de l'obésité infantile au Canada.

\section{Remerciements}

Rachel Prowse a bénéficié d'un soutien financier du programme pancanadien de la Fondation des maladies du cœur et de l'AVC du Canada et de la CIHR Training Grant in Population Intervention for Chronic Disease Prevention (programme PICDP, en anglais seulement, subvention $\mathrm{n}^{0}$ 53893); d'une bourse de recherche au doctorat des Instituts de recherche en santé du Canada (IRSC), soit la Bourse d'études supérieures du Canada Frederick Banting et Charles Best; et d'une bourse d'études au doctorat de l'Institut de recherche sur la santé des femmes et des enfants (WCHRI) offerte par la Fondation de l'Hôpital pour enfants Stollery. Rachel Prowse aimerait également exprimer sa gratitude au programme PICDP pour cette possibilité d'apprentissage par l'expérience qui lui a permis de collaborer avec un organisme tiers pour l'élaboration des objectifs de l'étude. L'auteure aimerait remercier la $\mathrm{D}^{\text {re }}$ Kim Raine pour son aide dans la préparation du manuscrit.

\section{Conflit d'intérêts}

Rachel Prowse n'a aucun lien financier susceptible de conduire à un conflit d'intérêts.

\section{Références}

1. Gluckman P, Nishtar S, Armstrong T. Ending childhood obesity: a multidimensional challenge. Lancet. 2015;385 (9973):1048-1050. doi: 10.1016/S0140 $-6736(15) 60509-8$
2. Halfon N, Larson K, Lu M, Tullis E, Russ S. Lifecourse health development: past, present and future. Matern Child Health J. 2014;18(2):344-365. doi: $10.1007 /$ s10995-013-1346-2

3. Organisation mondiale de la santé (OMS). Charte d'Ottawa pour la promotion de la santé - Première conférence internationale sur la promotion de la santé, Ottawa, 21 novembre 1986 [Internet]. Genève : OMS; 1986. En ligne à : http://www.euro.who.int /_data/assets/pdf_file/0003/129675 /Ottawa_Charter_F.pdf?ua = 1\&ua $=1$

4. Organisation mondiale de la santé (OMS). Le marketing des aliments à haute teneur en matières grasses, en sel et en sucre ciblant les enfants : bilan pour 2012-2013. Copenhague : Bureau régional de l'OMS pour l'Europe; 2013.

5. Lee NR, Kotler P. Social marketing: influencing behaviors for good. 4e éd. Thousand Oaks (CA) : Sage; 2011.

6. Cairns G, Angus K, Hastings G, Caraher M. Systematic reviews of the evidence on the nature, extent and effects of food marketing to children. A retrospective summary. Appetite. 2013;62:209-215. doi: 10.1016/j.appet .2012.04.017

7. Statistique Canada. Enquête sur la santé dans les collectivités canadiennes - Nutrition (2004). Ottawa (Ont.) : Statistique Canada; 2004.

8. Garriguet D. Nutrition : résultats de l'Enquête sur la santé dans les collectivités canadiennes - Vue d'ensemble des habitudes alimentaires des Canadiens 2004 [Internet]. Ottawa (Ont.) : Statistique Canada; 2004. En ligne à : http://www.statcan.gc.ca /pub/82-620-m/82-620-m2006002-fra .pdf

9. Organisation mondiale de la santé (OMS). A framework for implementing the set of recommendations on the marketing of foods and non-alcoholic beverages to children. Genève : OMS; 2012.

10. Office de la protection du consommateur. Publicité destinée aux enfants de moins de 13 ans : Guide d'application des articles 248 et 249 de la Loi sur la protection du consommateur. Québec (Québec) : Gouvernement du Québec; 2012.
11. Les normes canadiennes de la publicité. Initiative canadienne pour la publicité sur les aliments et boissons destinée aux enfants [Internet]. Toronto (Ont.) : Les normes canadiennes de la publicité (sans date) [consultation le 28 août 2015]. En ligne à : http://www .adstandards.com/fr/initiativeenfants /default.htm

12. Les normes canadiennes de la publicité. Le Code de la publicité radiotélévisée destinée aux enfants. Toronto (Ont.) : Les normes canadiennes de la publicité; 2015 [consultation le 10 août 2015]. En ligne à : http://www .adstandards.com/fr/clearance/childrens /broadcastCodeFor AdvertisingTo Children-TheCode.aspx

13. Santé Canada. Stratégie de Santé Canada en matière de saine alimentation [Internet]. Ottawa (Ont.) : Santé Canada; 2016 [consultation le 30 octobre 2016]. En ligne à : https://www .canada.ca/fr/services/sante/campagnes / vision-canada-en-sante/saine -alimentation.html

14. Dooris M. Holistic and sustainable health improvement: the contribution of the settings-based approach to health promotion. Perspect Public Health. 2009;129(1):29-36. doi: 10.1177 /1757913908098881.

15. Les normes canadiennes de la publicité. Initiative canadienne pour la publicité sur les aliments et boissons destinée aux enfants : Rapport de conformité 2014. Toronto (Ont.) : Les normes canadiennes de la publicité; 2015.

16. Edgar T, Huhman M, Miller GA. Understanding "place" in social marketing: a systematic review. Soc Mar Q. 2015;21(4):230-248. doi: 10.1177 $/ 1524500415607453$.

17. Santé Canada. Webinar on marketing to children. Documentation du webinaire en anglais présenté en ligne le 28 février 2017.

18. Whitelaw, S, Baxendale A, Bryce C, MacHardy L, Young I, Witney E. "Settings" based health promotion: a review. Health Promot Int. 2001;16(4): 339-353. doi:10.1093/heapro/16.4.339. 
19. Les normes canadiennes de la publicité. Initiative canadienne pour la publicité sur les aliments et boissons destinée aux enfants : Livre blanc sur les critères nutritionnels uniformes. Toronto (Ont.) : Les normes canadiennes de la publicité; 2014.

20. Les normes canadiennes de la publicité. Le Code canadien des normes de la publicité [Internet]. Toronto (Ont.) : Les normes canadiennes de la publicité; 2015 [consultation le 10 août 2015]. En ligne à : http:// www.adstandards.com/fr/standards /theCode.aspx

21. Gouvernement de la ColombieBritannique. Guidelines for food and beverage sales in BC schools. Victoria (Colombie-Britannique) : Gouvernement de la Colombie-Britannique; 2013.

22. Gouvernement de la ColombieBritannique. The guidelines for food and beverage sales in B.C. schools [Internet]. Victoria (Colombie-Britannique) : Gouvernement de la Colombie-Britannique; 2005 [mise à jour 2013; consultation le 11 avril 2017]. En ligne à : http://www2 .gov.bc.ca/gov/content/education -training/administration/legislation -policy/public-schools/the-guidelines -for-food-and-beverage-sales-in-bc -schools

23. Ministère de l'Éducation de l'Ontario. Politique/Programmes Note $\mathrm{n}^{\circ} 150$ : Politique concernant les aliments et les boissons dans les écoles [Internet]. Toronto (Ontario) : Imprimeur de la Reine pour l'Ontario [4 octobre 2010; consultation le 11 avril 2017]. En ligne à : http://www.edu.gov.on.ca/extra /fre/ppm/150.html

24. Gouvernement du Nouveau-Brunswick. Nutrition et amélioration de l'alimentation en milieu scolaire, Politique 711 [Internet]. Gouvernement du NouveauBrunswick; 2008. En ligne à : http:// www2.gnb.ca/content/dam/gnb /Departments/ed/pdf/K12/policies -politiques/f/711F.pdf

25. Ministère de l'Éducation du NouveauBrunswick. Nutrition et amélioration de l'alimentation en milieu scolaire : guide sur la Politique 711. Fredericton (Nouveau-Brunswick) : Ministère de l’Éducation; 2008.
26. Ministère de l'Éducation de la Nouvelle-Écosse. Food and nutrition policy for Nova Scotia public schools: policy directives and guidelines. Ministère de l'Éducation de la Nouvelle-Écosse; 2006.

27. Gouvernement de l'Île-du-PrinceÉdouard. School nutrition (for all grade levels - K-12) [Internet]. Règlement administratif du district scolaire de l'Est. Charlottetown (Îledu-Prince-Édouard) : Gouvernement de l'Île-du-Prince-Édouard; 2011. En ligne à : https://www.princeedwardisland .ca/sites/default/files/publications /english_schools_nutrition_policy.pdf

28. Gouvernement de la Saskatchewan. Healthy foods for my school: nutrition standards for Saskatchewan schools. Regina (Saskatchewan) : Gouvernement de la Saskatchewan; 2014.

29. Ministère de l'Éducation de la Saskatchewan. Nourishing minds: towards comprehensive school community health: nutrition policy development in Saskatchewan schools. Ministères de l'Éducation, de la Santé et des Services sociaux; 2009 [mise à jour 2012].

30. Gouvernement du Manitoba. Moving forward with school nutrition guidelines. Winnipeg (Manitoba) : Gouvernement du Manitoba; 2014.

31. Gouvernement du Manitoba. Loi sur l'administration scolaire : C.P.L.M. c. E10. Winnipeg (Manitoba) : Gouvernement du Manitoba; 2017.

32. Gouvernement de Terre-Neuve-etLabrador. School food guidelines for school food providers. 2e éd. St. John's (Terre-Neuve) : Gouvernement de Terre-Neuve-et-Labrador; 2009.

33. Gouvernement du Yukon. School nutrition policy no. 1025 [Internet]. Whitehorse (Yukon) : Gouvernement du Yukon; 2008. En ligne à : http:// www.education.gov.yk.ca/pdf/policies /school_nutrition_policy.pdf

34. Kelly B, Halford JC, Boyland EJ, et al. Television food advertising to children: a global perspective. Am J Public Health. 2010;100(9):1730-1736. doi: 10.2105/AJPH.2009.179267
35. Brierley M, Elliott C. Boys' healthy packaged food choices. Int $\mathrm{J}$ Mens Health. 2015;14(1):21-37.

36. Elliott C. Food as people: teenagers' perspectives on food personalities and implications for healthy eating. Soc Sci Med. 2014;121:85-90. doi:10.3149/jmh .1401 .21

37. Elliott C. Packaging health: Examining "better-for-you" foods targeted at children. Can Public Policy. 2012;38(2): 265-281. doi: 10.3138/cpp.38.2.265

38. Lebel E, Hamelin A-M, Lavallée M, Bédard A, Dubé A. Publicité télévisée sur les aliments visant les enfants québécois. Communication. 2005;24(1): 65-85. doi: 10.4000/communication .3259

39. Laperrière J-P. Analyse comparative de la forme des messages publicitaires pouvant s'adresser aux enfants. Montréal : Université du Québec à Montréal; 2009.

40. Elliott C. "It's junk food and chicken nuggets": children's perspectives on 'kids' food and the question of food classification. J Consumer Behav. 2011;10(3):133-140. doi: 10.1002/cb .360

41. Adams J, Hennessy-Priest K, Ingimarsdóttir S, Sheeshka J, Ostbye $\mathrm{T}$, White $\mathrm{M}$. Changes in food advertisements during 'prime-time' television from 1991 to 2006 in the UK and Canada. Br J Nutr. 2009;102(4):584593. doi: $10.1017 /$ S0007114509220848

42. Adams J, Hennessy-Priest K, Ingimarsdóttir S, Sheeshka J, Ostbye T, White M. Food advertising during children's television in Canada and the UK. Arch Dis Child. 2009;94(9): 658-662.

43. Potvin Kent M, Dubois L, Wanless A. Food marketing on children's television in two different policy environments. Int J Pediatr Obes. 2011; 6(2Part2):e433-e441. doi: 10.3109 /17477166.2010.526222.

44. Potvin Kent M, Dubois L, Wanless A. A nutritional comparison of foods and beverages marketed to children in two advertising policy environments. Obesity. 2012;20(9):1829-1837. doi: 10.1038/oby.2011.161. 
45. Potvin Kent M, Dubois L, Wanless A. Self-regulation by industry of food marketing is having little impact during children's preferred television. Int J Pediatr Obes. 2011;6(5-6):401-408. doi: 10.3109/17477166.2011.606321.

46. Potvin Kent M, Martin CL, Kent EA. Changes in the volume, power and nutritional quality of foods marketed to children on television in Canada. Obesity (Silver Spring). 2014;22(9): 2053-2060. doi:10.1002/oby.20826.

47. Potvin Kent M, Wanless A. The influence of the Children's Food and Beverage Advertising Initiative: change in children's exposure to food advertising on television in Canada between 2006-2009. Int J Obes. 2014;38(4):558562. doi: 10.1038/ijo.2014.4.

48. Hudson S, Elliott C. Measuring the impact of product placement on children using digital brand integration. J Food Prod Mark. 2013;19(3):176-200. doi: 10.1080/10454446.2013.724370.

49. Brady J, Farrell A, Wong S, Mendelson R. Beyond television: children's engagement with online food and beverage marketing. Clin Med Insights: Pediatr [Internet]. 2008;2:1-9. En ligne à : http://insights.sagepub.com/beyond -television-childrens-engagement-with -online-food-and-beverage-m-article -a916

50. Brady J, Mendelson R, Farrell A, Wong $S$. Online marketing of food and beverages to children: a content analysis. Can J Diet Pract Res. 2010;71(4):166171. doi: 10.3148/71.4.2010.166.

51. Potvin Kent M, Dubois L, Kent E, Wanless A. Internet marketing directed at children on food and restaurant websites in two policy environments. Obesity (Silver Spring). 2013;21(4): 800-807. doi: 10.1002/oby.20124.

52. Velazquez CE, Black JL, Ahmadi N. Food and beverage promotions in Vancouver schools: a study of the prevalence and characteristics of inschool advertising, messaging, and signage. Prev Med Rep. 2015;2:757764. doi: 10.1016/j.pmedr.2015.08.020.

53. Berry B, McMullen T. Visual communication to children in the supermarket context: Health protective or exploitive? Agric Human Values. 2008;25(3):333-348. doi:10.1007/s10460 $-007-9110-0$.
54. Elliott C. Assessing "fun foods": nutritional content and analysis of supermarket foods targeted at children. Obes Rev. 2008;9(4):368-377. doi: 10.1111/j.1467-789X.2007.00418.x.

55. Elliott C. Packaging fun: analyzing supermarket food messages targeted at children. Can J Commun. 2012; 37(2):303-318. doi: $10.22230 / \mathrm{cjc}$ .2012v37n2a2550.

56. Murray C. Examining the nutritional content of prepackaged foods and beverages marketed to children in Canada [mémoire de maîtrise]. Toronto (Ont.) : Université de Toronto; 2014.

57. Elliott C. Healthy food looks serious: how children interpret packaged food products. Can J Commun. 2009;34(3). doi: 10.22230/cjc.2009v34n3a2220.

58. Elliott C, Brierley M. Healthy choice?: Exploring how children evaluate the healthfulness of packaged foods. Can J Public Health. 2012;103(6):e453-e458. doi: 10.17269/cjph.103.3419.

59. Hobin EP, Hammond DG, Daniel S, Hanning RM, Manske S. The Happy Meal ${ }^{\circledR}$ effect: the impact of toy premiums on healthy eating among children in Ontario, Canada. Can J Public Health. 2012;103(4):e244-e248. doi: 10.17269/cjph.103.3181.

60. Elliott C, Den Hoed R, Conlon M. Food branding and young children's taste preferences: a reassessment. Can J Public Health. 2013;104(5):e364-e368. doi: 10.17269/cjph.104.3957.

61. Boyland EJ, Nolan S, Kelly B, et al. Advertising as a cue to consume: a systematic review and meta-analysis of the effects of acute exposure to unhealthy food and nonalcoholic beverage advertising on intake in children and adults. Am J Clin Nutr. 2016; 103(2):519-533. doi: 10.3945/ajen.115 .120022 .

62. Norman J, Kelly B, Boyland E, McMahon A-T. The impact of marketing and advertising on food behaviours: evaluating the evidence for a causal relationship. Curr Nutr Rep. 2016;5(3):139-149. doi: 10.1007/s13668 $-016-0166-6$
63. Ohri-Vachaspati P, Isgor Z, Rimkus L, Powell LM, Barker DC, Chaloupka FJ. Child-directed marketing inside and on the exterior of fast food restaurants. Am J Prev Med. 2015;48(1):22-30. doi: 10.1016/j.amepre.2014.08.011.

64. Kelly B, Bauman AE, Baur LA. Population estimates of Australian children's exposure to food and beverage sponsorship of sports clubs. J Sci Med Sport. 2014;17(4):394-398. doi: 10.1016/j.jsams.2013.07.005.

65. Kelly B, Cretikos M, Rogers K, King L. The commercial food landscape: outdoor food advertising around primary schools in Australia. Aust Nz J Publ Heal. 2008;32(6):522-528. doi: 10.1111 /j.1753-6405.2008.00303.x.

66. Dhar T, Baylis K. Fast-food consumption and the ban on advertising targeting children: the Quebec experience. J Mark Res. 2011;48(5):799-813. doi: 10.1509/jmkr.48.5.799

67. Froese-Germain B, Hawkey C, Larose A, McAdie P, Shaker E. Commercialism in Canadian schools: who's calling the shots? Ottawa (Ont.) : Fédération canadienne des enseignantes et des enseignants; 2006.

68. Leibowitz J, Rosch J, Ramirez E, Brill J, Ohlhausen M. A review of food marketing to children and adolescents: follow-up report. Washington (DC) : Commission fédérale du commerce des États-Unis; 2012.

69. Statistique Canada. Enquête sociale générale - 2010 : Aperçu sur l'emploi du temps des Canadiens - Faits saillants [Internet]. Ottawa (Ont.) : Statistique Canada; 2011 [mise à jour le 12 juillet 2011; consultation le 22 septembre 2016]. En ligne à : http://www.statcan.gc.ca/pub/89-647 -x/2011001/hl-fs-fra.htm

70. Association canadienne d'éducation. The 2012/2013 school calendar. Toronto (Ont.) : Association canadienne d'éducation; 2013.

71. Canadian Grocer. Grocers should look at 'fresh' opportunities in 2013: Nielsen [Internet]. Toronto (Ont.) : Canadian Grocer; 2013. En ligne à : http://www .canadiangrocer.com/top-stories/grocers -should-look-at-fresh-opportunities-in -2013-nielsen-20506 
72. Galbraith-Emami S, Lobstein T. The impact of initiatives to limit the advertising of food and beverage products to children: a systematic review. Obes Rev. 2013;14(12):960-974. doi:10.1111 /obr. 12060

73. Raine KD, Lobstein T, Landon J, et al. Restricting marketing to children: consensus on policy interventions to address obesity. J Public Health Policy. 2013;34(2):239-253. doi:10.1057/jphp .2013 .9

74. Lloyd-Williams F, Bromley H, Orton L, et al. Smorgasbord or symphony? Assessing public health nutrition policies across 30 European countries using a novel framework. BMC Public Health [Internet]. 2014;14:1195. En ligne à : http://dx.doi.org/10.1186/1471 -2458-14-1195

75. Palakshappa D, Fiks AG, Faerber JA, et Feudtner C. Association between state school nutrition laws and subsequent child obesity. Prev Med. 2016; 90:107-113. doi: 10.1016/j.ypmed.2016 .06 .039

76. Agence de la santé publique du Canada (ASPC). Freiner l'obésité juvénile : Cadre d'action fédéral, provincial et territorial pour la promotion du poids santé. Ottawa (Ont.) : ASPC; 2011.

77. Statistique Canada. CANSIM (base de données) : Tableau 051-0001 : Estimations de la population, selon le groupe d'âge et le sexe au 1er juillet, Canada, provinces et territoires [Internet]. Ottawa (Ont.) : Statistique Canada [mise à jour le 28 septembre 2016; consultation le 12 juin 2017]. En ligne à : http://www5.statcan.gc.ca/cansim /a26;jsessionid = F7F0A62FF58A9473 BA738B3F23780418? id = 510001\&lang $=$ fra\&retrLang $=$ fra

78. Statistique Canada. CANSIM (base de données) : Tableau 102-4512 : Naissances vivantes, selon les semaines de gestation et le sexe, Canada, provinces et territoires [Internet]. Ottawa (Ont.) : Statistique Canada [mise à jour le 25 octobre 2016; consultation le 12 juin 2017]. En ligne à : http:// www5.statcan.gc.ca/cansim/a26?id $=1024512$ \&lang $=$ fra\&retrLang $=$ fra
79. Ministère de l'Éducation, du Loisir et du Sport. Pour un virage santé à l'école [Internet]. Québec (Québec): Gouvernement du Québec; 2007. En ligne à : http://www.education.gouv .qc.ca/fileadmin/site_web/documents /dpse/adaptation_serv_compl /virageSanteEcole_PolCadre.pdf 